\title{
WNT6/ACC2-induced storage of triacylglycerols in macrophages is exploited by Mycobacterium tuberculosis
}

\author{
Julius Brandenburg, ${ }^{1,2}$ Sebastian Marwitz,, ${ }^{3,4}$ Simone C. Tazoll, ${ }^{1}$ Franziska Waldow, ${ }^{2,5}$ Barbara Kalsdorf, ${ }^{2,6}$ Tim Vierbuchen, \\ Thomas Scholzen, ${ }^{8}$ Annette Gross, ${ }^{1}$ Svenja Goldenbaum, ${ }^{1}$ Alexandra Hölscher, ${ }^{9}$ Martina Hein, ${ }^{8}$ Lara Linnemann, ${ }^{10}$ \\ Maja Reimann, ${ }^{6}$ Andreas Kispert, ${ }^{11}$ Michael Leitges, ${ }^{12}$ Jan Rupp, ${ }^{2,13}$ Christoph Lange, ${ }^{2,6,14,15}$ Stefan Niemann, ${ }^{2,16}$ Jochen Behrends, ${ }^{8}$ \\ Torsten Goldmann, ${ }^{3,4}$ Holger Heine, ${ }^{7}$ Ulrich E. Schaible, ${ }^{2,10}$ Christoph Hölscher, ${ }^{2,9}$ Dominik Schwudke, ${ }^{2,4,5}$ and Norbert Reiling, \\ ${ }^{1}$ Microbial Interface Biology, Research Center Borstel, Leibniz Lung Center, Borstel, Germany. ${ }^{2}$ Cerman Center for Infection Research (DZIF), Site Hamburg-Lübeck-Borstel-Riems, Hamburg, Cermany. \\ ${ }^{3}$ Pathology, Research Center Borstel, Borstel, Germany. ${ }^{4}$ Airway Research Center North (ARCN), Member of the German Center for Lung Research (DZL), Grosshansdorf, Germany. ${ }^{5}$ Bioanalytical Chemistry, \\ ${ }^{6}$ Clinical Infectious Diseases, ${ }^{7}$ Innate Immunity, ${ }^{8}$ Fluorescence Cytometry Core Unit, ${ }^{9}$ Infection Immunology, and ${ }^{10}$ Cellular Microbiology, Research Center Borstel, Borstel, Germany. ${ }^{11}$ Institute for Molecular \\ Biology, Hannover Medical School, Hannover, Germany. ${ }^{2}$ Division of BioMedical Sciences/Faculty of Medicine, Memorial University of Newfoundland, St. John's, Newfoundland, Canada. ${ }^{13}$ Department of \\ Infectious Diseases and Microbiology and ${ }^{14}$ Respiratory Medicine \& International Health, University of Lübeck, Lübeck, Germany. ${ }^{15}$ Baylor College of Medicine and Texas Children's Hospital, Houston, Texas, \\ USA. ${ }^{16}$ Molecular and Experimental Mycobacteriology, Research Center Borstel, Borstel, Germany.
}

\begin{abstract}
In view of emerging drug-resistant tuberculosis (TB), host-directed adjunct therapies are urgently needed to improve treatment outcomes with currently available anti-TB therapies. One approach is to interfere with the formation of lipid-laden "foamy" macrophages in the host, as they provide a nutrient-rich host cell environment for Mycobacterium tuberculosis (Mtb). Here, we provide evidence that Wnt family member 6 (WNT6), a ligand of the evolutionarily conserved Wingless/ Integrase 1 (WNT) signaling pathway, promotes foam cell formation by regulating key lipid metabolic genes including acetylCoA carboxylase 2 (ACC2) during pulmonary TB. Using genetic and pharmacological approaches, we demonstrated that lack of functional WNT6 or ACC2 significantly reduced intracellular triacylglycerol (TAC) levels and Mtb survival in macrophages. Moreover, treatment of Mtb-infected mice with a combination of a pharmacological ACC2 inhibitor and the anti-TB drug isoniazid (INH) reduced lung TAG and cytokine levels, as well as lung weights, compared with treatment with INH alone. This combination also reduced Mtb bacterial numbers and the size of mononuclear cell infiltrates in livers of infected mice. In summary, our findings demonstrate that Mtb exploits WNT6/ACC2-induced storage of TAGs in macrophages to facilitate its intracellular survival, a finding that opens new perspectives for host-directed adjunctive treatment of pulmonary TB.
\end{abstract}

\section{Introduction}

Tuberculosis (TB) is the leading cause of death from a single bacterial agent (1). The current increase in numbers of patients affected by multidrug-resistant and rifampicin-resistant TB (2) severely jeopardizes control of the TB epidemic as envisaged by the WHO End TB strategy (1). A novel and innovative approach to fight disease without incurring the risk of bacterial resistance development is to target host factors that facilitate Mycobacterium tuberculosis (Mtb) survival (3).

As an intracellular pathogen, Mtb has evolved to reside within the hostile environment of macrophages (4). These cells serve as the main host cell for Mtb but are also able to restrict infection when appropriately activated. In response to signals such

Conflict of interest: N. Reiling and J. Brandenburg have filed a patent application titled "ACC inhibitors as means and methods for treating mycobacterial diseases" (W02018007430A1)

Copyright: ( 2021, American Society for Clinical Investigation.

Submitted: July 6, 2020; Accepted: July 6, 2021; Published: August 16, 2021.

Reference information: J Clin Invest. 2021;131(16):e141833.

https://doi.org/10.1172/JCl141833. as hypoxia (5), microbial structures (6), and Mtb infection (7), macrophages undergo a substantial metabolic shift away from oxidative metabolism toward glycolysis. Rewiring of cellular metabolism is necessary to mediate macrophage activation (8) and proinflammatory polarization (9) and to control Mtb growth $(10,11)$. These activating signals, however, also promote the accumulation of neutral lipids in macrophages as fatty acid oxidation is downregulated $(12,13)$.

Macrophages with a "foamy," neutral lipid-rich phenotype are abundantly found in the Mtb-infected human lung and particularly in TB granulomas (14). Foamy macrophages accumulate neutral lipids, triacylglycerols (TAGs), and cholesterol esters (CEs) in cytoplasmic compartments termed lipid droplets. Intracellular Mtb is found in close proximity to lipid droplets (15), and the utilization of fatty acids (16) and cholesterol (17) by Mtb was shown to be critical for its virulence and persistence in vivo. Moreover, foamy macrophages have been associated with progressive TB pathology due to a temporal and spatial correlation between the death of foamy macrophages and granuloma evolvement toward tissue necrosis ultimately leading to the release of mycobacteria into the airways (14). Thus, interfering with foam cell formation 

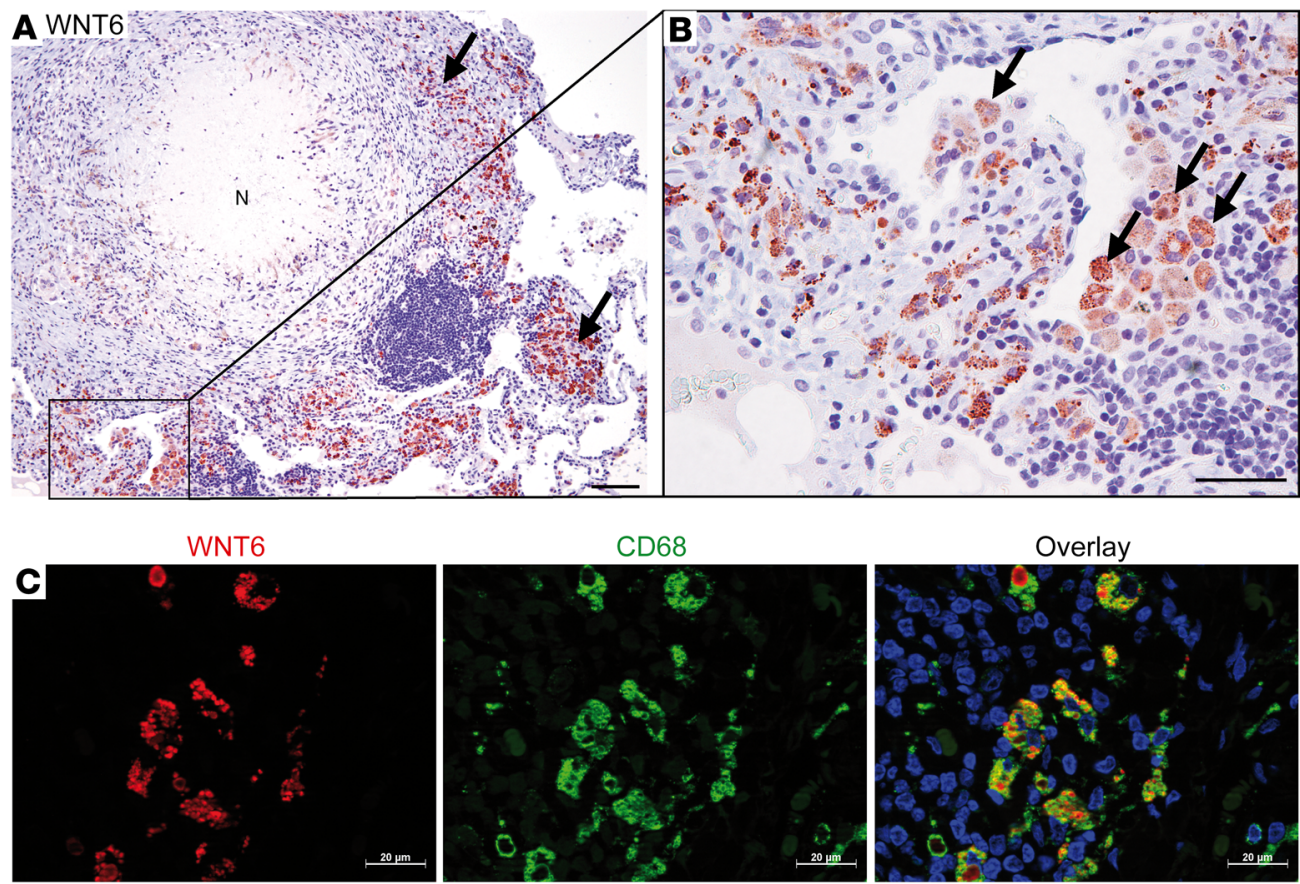

Figure 1. WNT6 is expressed in foamy macrophages during pulmonary TB. (A and B) Immunohistochemical detection of WNT6 in a lung tissue section of a TB patient. Black arrows in $\mathbf{A}$ indicate areas of WNT6-expressing cells, and those in B indicate cells with a foamy morphology. N, necrosis. (C) Immunofluorescence detection of WNT6 (red), the macrophage/monocyte marker CD68 (green), and cell nuclei (blue, DAPI) in a lung tissue section from a TB patient (same patient as in $\mathbf{A}$ and B). (D) Immunofluorescence staining for WNT6 (red), neutral lipids (green, BODIPY 493/503), and cell nuclei (blue, DAPI) in a lung tissue section derived from an Mtb-infected ( 200 CFUs) IL-13-overexpressing mouse (day 63 p.i.). Representative observations from 3 independent patients $(\mathbf{A}-\mathbf{C})$ and experiments $(\mathbf{D})$ are shown. Scale bars: (A) $100 \mu \mathrm{m}$; (B) $50 \mu \mathrm{m}$; (C and D) $20 \mu \mathrm{m}$.
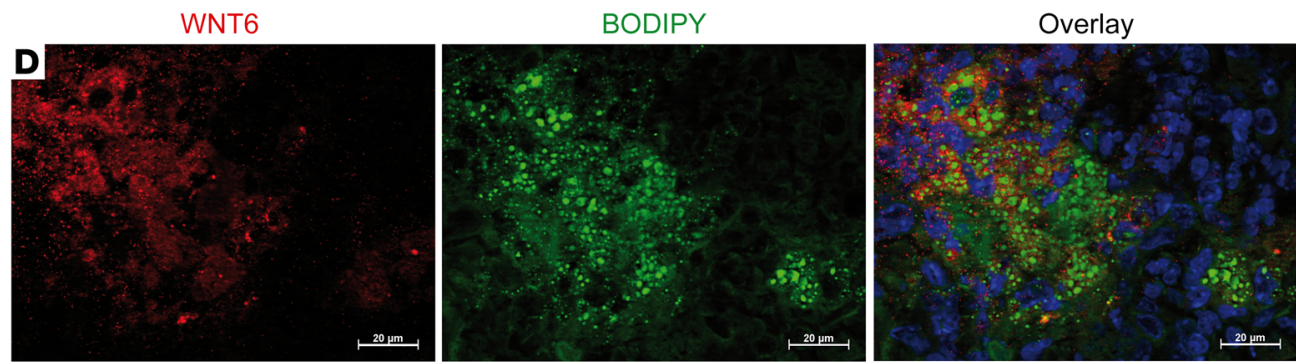

during infection may deprive Mtb of essential nutrients within its intracellular niche and may restrict bacterial dissemination.

The Wingless/Integrase 1 (WNT) signaling pathway, which is evolutionarily highly conserved in multicellular eukaryotic organisms, comprises 19 extracellular WNT ligands in humans and mice (18). WNT signaling regulates basic processes such as proliferation, differentiation, and death in virtually all cells, including immune cells (19). We previously demonstrated that Mtb infection induces expression of Wnt family member 6 (WNT6) in macrophages, which acts as an antiinflammatory feedback regulator dampening responses to mycobacteria (20). In the current study, we uncover a role for WNT6 in macrophage metabolism, showing that WNT6 drives foam cell formation during Mtb infection. We provide evidence that WNT6-induced acetyl-CoA carboxylase 2 (ACC2) activity in macrophages and mice promotes storage of TAG, which is utilized by Mtb to facilitate intracellular survival.

\section{Results}

WNT6 is expressed in foamy macrophages during pulmonary TB. We have previously reported that WNT6 is expressed in granulomatous infiltrations in the lungs of C57BL/6 mice experimentally infected with Mtb (20). To extend this observation to human pulmonary TB, we stained lung tissue samples of 3 independent TB patients, who had undergone resection of infected lung tis- sue, with an antibody directed against WNT6 (Figure 1 and Supplemental Figure 1, A-C; supplemental material available online with this article; https://doi.org/10.1172/JCI141833DS1). WNT6 protein expression was found in cells within nascent granulomas but also in the periphery of necrotizing granulomas (black arrows, Figure 1A). We found WNT6 expression almost exclusively in cells positive for the monocyte/macrophage marker CD68 (Figure 1C and Supplemental Figure 1, A and B). Thus, WNT6 protein expression during Mtb infection in humans is restricted to cells of the myeloid lineage, corroborating previous observations in mice (20). Notably, WNT6 was prominently expressed in cells with a foam cell morphology (black arrows, Figure 1B). Consistent with that, cells strongly expressing WNT6 (Supplemental Figure 1D) also showed prominent staining for the lipid droplet scaffolding protein perilipin 2 (PLIN2) (Supplemental Figure 1E).

Next, we stained lung tissue sections of Mtb-infected IL-13overexpressing mice, which are known to develop a human-like pathology upon Mtb infection including centrally necrotizing granulomas with an adjacent zone of foamy macrophages containing numerous lipid droplets (Supplemental Figure 2, A and B, and ref. 21). We observed an intense WNT6 expression (Figure 1D, left panel, red) in areas of prominent neutral lipid accumulation as visualized by staining with the neutral lipid dye BODIPY 493/503 (22) (Figure 1D, middle panel, green; and Supplemental Figure 
A

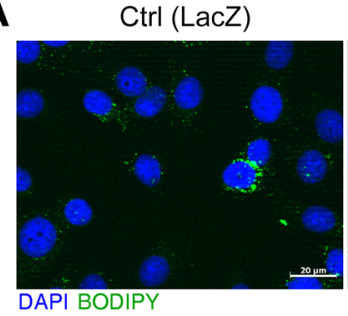

D

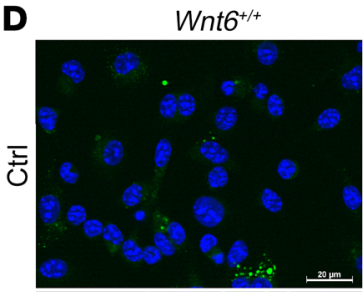

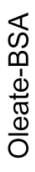

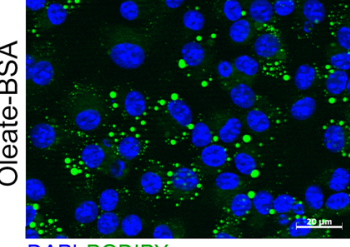

DAPI BODIPY

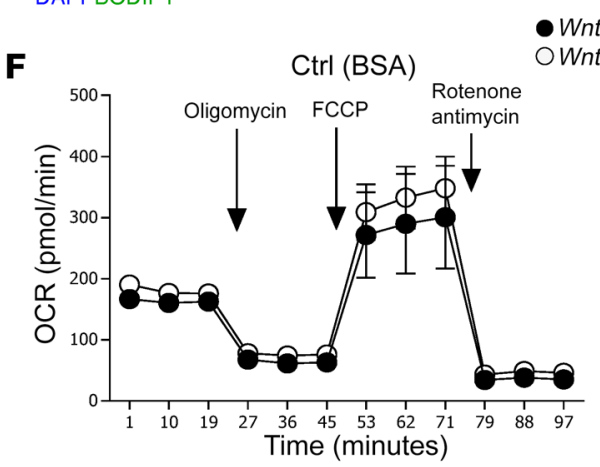

G
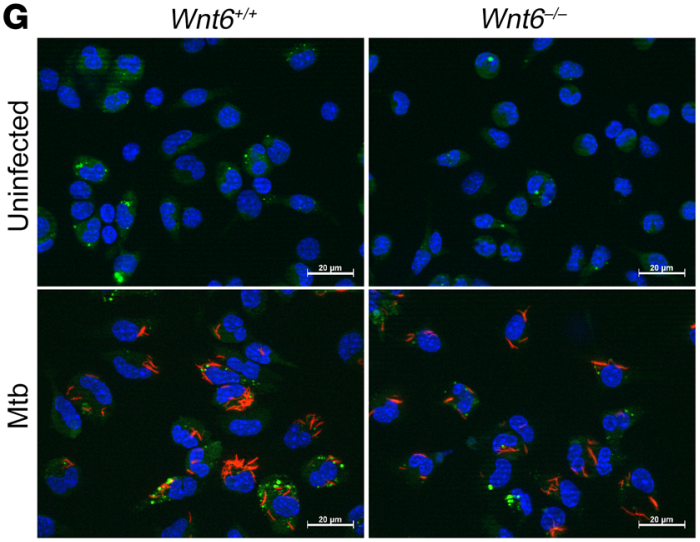

DAPI BODIPY Cherry-Mtb

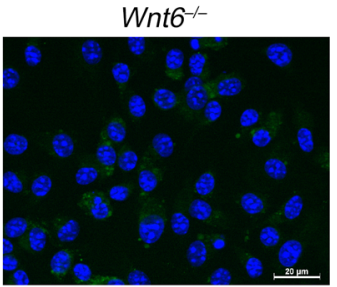

WNT6
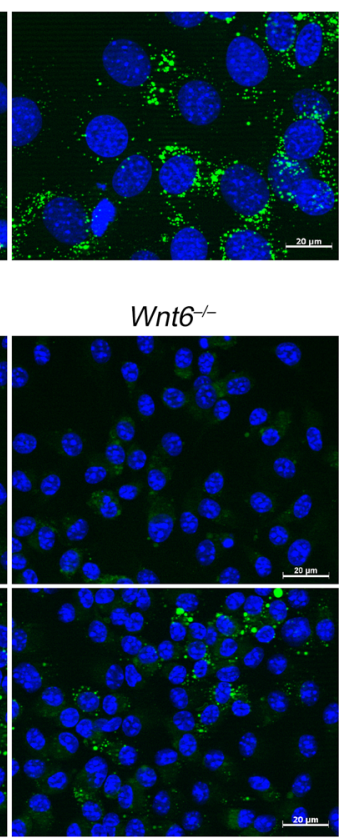

Wnt6 $6^{+/+}$
B

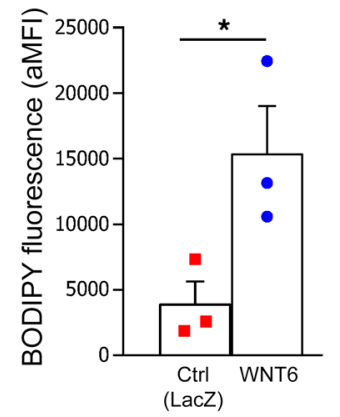

E

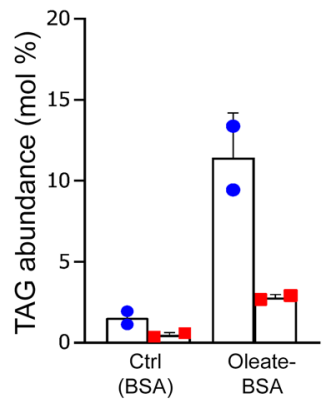

Oleate-BSA

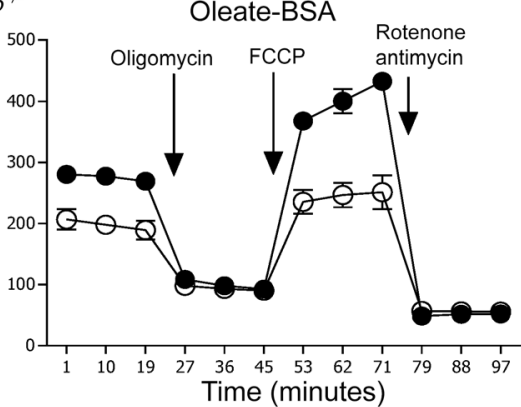

H

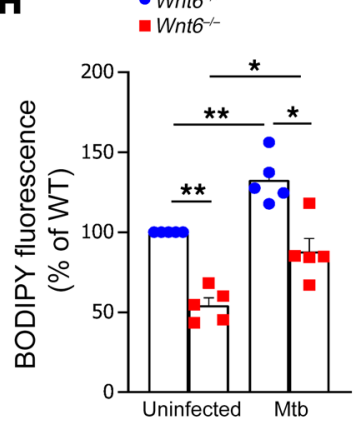

C

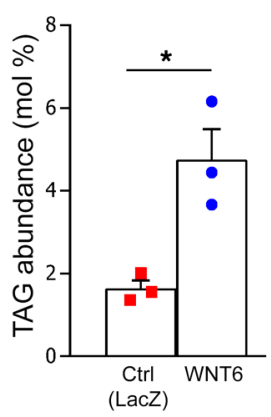

Figure 2. WNT6 drives the accumulation of TAG-rich lipid droplets. (A) Visualization of neutral lipids in WNT6-overexpressing (WNT6) or control (LacZ) NIH 3T3 cells by fluorescence microscopy. Nuclei (blue, DAPI); neutral lipids (green, BODIPY 493/503). A representative staining from 2 independent experiments is shown. (B) Quantification of neutral lipids by flow cytometry (same cells as in $\mathbf{A}$; arithmetic mean fluorescence intensity [aMFI] of BODIPY signals); $n=3$. (C) Mass spectrometry-based quantification of TAGs (same cells as in $\mathbf{A}$ ); $n=$ 3. (D) Visualization of neutral lipids in BMDMs from $\mathrm{Wnt6}^{+/+}$or Wnt6 ${ }^{-/-}$mice incubated for 24 hours in the absence (Ctrl) or presence of oleic acid (oleate-BSA, $200 \mu \mathrm{M}$ ) by fluorescence microscopy. A representative staining from 2 independent experiments is shown. (E) Mass spectrometry-based quantification of TAGs in $\mathrm{BMDMs}$ from $\mathrm{Wnt6}^{+/+}$or Wnt6 ${ }^{-/-}$mice incubated for 24 hours in the absence (Ctrl, BSA) or presence of oleic acid (oleate-BSA, $200 \mu \mathrm{M}$ ); $n=2$. (F) Oxygen consumption rate (OCR) of BMDMs incubated for 24 hours in the absence (Ctrl, BSA) or presence of oleic acid (oleateBSA, $200 \mu \mathrm{M}$ ) as determined on an extracellular flux analyzer; $n=2$. ( $\mathbf{G}$ and $\mathbf{H}$ ) Visualization (G) and quantification (H) of neutral lipids in uninfected and Mtb-infected (MOI 0.1:1) $W_{n t 6^{+/+}}$and $W_{n t 6^{-/-}}$peritoneal macrophages after 24 hours by fluorescence microscopy. mCherry-Mtb (red); nuclei (blue, DAPI); neutral lipids (green, BODIPY); $n=5$. Statistical analyses were carried out using 1-way ANOVA with Holm-Šidák's multiple-comparison test. ${ }^{*} P \leq$ $0.05,{ }^{* *} P \leq 0.01$. Data are depicted as mean \pm SEM, except in $\mathbf{E}$, where mean $\pm \mathrm{SD}$ is shown. Scale bars: $20 \mu \mathrm{m}$.

2C). Taken together, our findings associate WNT6 with the presence of foamy macrophages in pulmonary TB.

WNT6 drives accumulation of TAG-rich lipid droplets. We hypothesized that WNT6 expression is functionally linked to the acquisition of a "foamy," lipid droplet-rich phenotype. To demonstrate this, we analyzed WNT6-overexpressing NIH 3T3 cells and visualized neutral lipids by use of BODIPY 493/503 (22). Fluorescence microscopic analysis revealed an enhanced number of neutral lipid-rich structures (Figure 2A, BODIPY, green) in
WNT6-overexpressing NIH 3T3 cells compared with control cells, a finding that was independently confirmed by flow cytometry (Figure 2B). Consistent with this, mass spectrometry-based lipid analysis revealed a significantly increased abundance of TAGs in WNT6-overexpressing cells compared with control cells (Figure 2C). In contrast, the abundance of phosphatidylcholines (PCs), as marker lipids of cellular membranes, remained unchanged (Supplemental Figure 3A), while CE levels were even decreased in WNT6-overexpressing cells (Supplemental Figure 3B). To extend 
these findings to macrophages, Mtb's main host cells, we next analyzed bone marrow-derived macrophages (BMDMs) from WNT6-competent $\left(W n 6^{+/+}\right)$and WNT6-deficient $\left(\right.$Wnt $\left.^{-/-}\right)$mice. Treatment of BMDMs with oleate-BSA, a conjugate of the dietary fatty acid oleic acid with the carrier protein BSA (22), induced lipid droplet formation and increased TAG abundance in $W n t 6^{+/+}$ BMDMs compared with untreated cells (Figure 2, D and E). Lipid droplets and TAG levels were strongly reduced in oleate-BSAtreated $W n t 6^{-/-}$macrophages compared with equally treated wildtype cells (Figure 2, D and E). PC levels, which increased upon oleate-BSA treatment (PC 36:2), remained comparable between $W_{n t 6^{+/+}}$and Wnt $^{-/-}$cells (Supplemental Figure 3C), supporting the notion that WNT6 specifically promotes synthesis of TAGrich lipid droplets.

As these results demonstrate that WNT6 regulates macrophage metabolism, we studied the influence of Wnt6 deficiency on mitochondrial activity using an extracellular flux analyzer. We observed similar oxygen consumption rates (OCRs) between $\mathrm{Wnt}^{+/+}$and Wnt $6^{-/-}$macrophages when cultivating them under control conditions (BSA, Figure 2F, left panel). However, basal as well as maximal respiration measured by the OCR were substantially increased in Wnt6-deficient cells when treated with oleate-BSA (Figure 2F, right panel). An enhanced respiratory metabolic activity in Wnt6-deficient cells upon fatty acid supplementation indicates that WNT6 inhibits mitochondrial fatty acid oxidation and thereby shifts fatty acid metabolism toward neutral lipid synthesis.

Next, we assessed whether WNT6 regulates neutral lipid metabolism in in vivo-differentiated macrophages in the absence or presence of Mtb. We isolated peritoneal macrophages from $W_{n t 6^{+/+}}$and $W n t 6^{-/-}$mice that were infected with mCherryexpressing Mtb for 24 hours and analyzed by fluorescence microscopy (Figure 2, G and H). Neutral lipid levels as determined by BODIPY 493/503 staining were significantly lower in uninfected cells from $\mathrm{Wnt}^{-/-}$mice compared with $\mathrm{Wnt} \mathrm{6}^{+/+}$mice, suggesting that disrupted WNT6 signaling affects neutral lipid levels under homeostatic conditions. Mtb infection - independently of the presence of WNT6 - enhanced the amounts of intracellular neutral lipids by about 30\% in comparison with uninfected cells, which is consistent with data from independent studies (23-25). A significant reduction of BODIPY fluorescence by about $45 \%$ was observed in both uninfected and Mtb-infected peritoneal macrophages from $\mathrm{Wnt}^{-/-}$mice compared with respective $\mathrm{Wnt6^{+/+ }}$ cells. Our data show that WNT6-dependent and WNT6-independent pathways contribute to the formation of lipid droplets in in vivodifferentiated macrophages. Together, our findings reveal a TAG storage-promoting effect of WNT6 signaling, in both the absence and the presence of Mtb.

WNT6 induces the expression of lipid metabolic enzymes critical for TAG synthesis and lipid droplet accumulation. To identify the cellular processes that are altered in the absence of Wnt6, we conducted a microarray-based gene expression analysis comparing Mtb-infected $W n t 6^{+/+}$and $W n t 6^{-/-}$macrophages (Gene Expression Omnibus [GEO] GSE160039). We infected cells with an MOI of 3:1 for 24 hours, because we previously observed that Mtb substantially induces Wnt6 expression in BMDMs under these conditions (20). By performing a gene set enrichment analysis (26) using gene sets from the peer-reviewed Reactome pathway data- base, we identified "Metabolism of lipids and lipoproteins" on rank 4 (FDR $q$ value, $2.58 \times 10^{-13}$ ) under the top 10 enriched gene sets (Supplemental Figure 4A) along with other expected sets of genes such as "Immune system," "Cell cycle," and "Developmental biology," which corroborate previous data (20). In-depth analysis revealed that genes encoding key factors involved in fatty acid uptake (27), activation (28), and mitochondrial oxidation were significantly upregulated in $\mathrm{Wnt6}^{-/-}$cells (Figure 3A). Notably, $\mathrm{Wnt}^{-/-}$cells showed a strong upregulation of Cpt1b, a gene encoding an isoform of carnitine palmitoyltransferase I (CPT1), the rate-limiting enzyme in mitochondrial $\beta$-oxidation (29). Consistent with these observations, $W n t 6^{-/-}$cells exhibited decreased expression of genes associated with fatty acid synthesis (30) (Figure 3B). Moreover, we observed a strongly reduced expression of acyl-CoA:diacylglycerol acyltransferase 2 (Dgat2), perilipin 3 (Plin3), and acetyl-CoA carboxylase 2 (Acacb, ACC2) in Wnt6 ${ }^{-1-}$ cells, which represent factors critically involved in TAG synthesis and lipid droplet formation (31-34).

Gene expression analysis based on quantitative reverse transcriptase PCR (RT-qPCR) revealed that Mtb infection dosedependently induced Acacb and Plin3 mRNA expression in $W n t 6^{+/+}$ macrophages, while mRNA levels remained at baseline in $W_{n t}{ }^{-1-}$ macrophages at all MOIs tested (Figure 3C and Supplemental Figure 4B). In contrast Cpt $1 b$ mRNA levels were significantly increased in $\mathrm{Wnt}^{-/-}$macrophages upon infection when compared with respective $\mathrm{Wnt}^{+/+}$cells (Figure 3D). Consistent with these findings, a strongly enhanced expression of Acacb, Dgat2, Plin2, and Plin3 was observed in WNT6-overexpressing (WNT6) NIH $3 \mathrm{~T} 3$ cells compared with control [Ctrl (LacZ)] cells (Figure 3E), providing further evidence that WNT6 drives the expression of factors critically involved in TAG synthesis (ACC2 [refs. 33, 34], DGAT2 [ref. 31]) and lipid droplet biogenesis (PLIN2, PLIN3) (32). Moreover, we treated human monocyte-derived macrophages (hMDMs) with WNT6 conditioned medium (WNT6 CM) or control conditioned medium (control CM) for 24 hours, which revealed that WNT6 induced $A C A C B$ and DGAT2 mRNA expression also in human macrophages (Figure $3 \mathrm{~F}$ ). Together, these data show that WNT6 drives the expression of key lipid metabolic enzymes, including ACC2 and DGAT2, in both murine and human cells.

WNT6-mediated changes in host lipid metabolism promote Mtb survival in macrophages. Next, we assessed whether WNT6 expression affects the development of intracellular bacterial numbers in Mtb-infected macrophages over time. Mtb CFU analysis in $\mathrm{Wnt6}^{+/+}$and $W n t 6^{-/-}$macrophages 4 hours postinfection (p.i.) revealed a comparable uptake of mycobacteria by both cell types independent of the infection dose used (Supplemental Figure 4C and Figure 3G). At day 3 p.i., however, intracellular bacterial loads were significantly reduced in cells lacking Wnt6, as indicated by approximately 2-fold (MOI 1:1) reduced Mtb CFUs in Wnt6 $^{-/-}$cells compared with $\mathrm{Wnt}^{+/+}$macrophages (Figure $3 \mathrm{G}$ ). This significant effect of Wnt6 deficiency on bacterial loads was also observed at day 7 p.i., as revealed by a 2.2-fold (MOI 0.5:1) and 3.3-fold (MOI 1:1) reduction of Mtb CFUs in $\mathrm{Wnt}^{-/-}$compared with $\mathrm{Wnt}^{+/+}$macrophages (Supplemental Figure $4 \mathrm{C}$ and Figure 3G). At both day 3 and day 7 p.i., the quantification of nitrite in cell culture supernatants of $\mathrm{Wnt}^{+/+}$and $\mathrm{Wnt6}^{-/-}$macrophages revealed similar production of nitric oxide, a well-established tuberculostatic host 
A Fatty acid uptake \& degradation
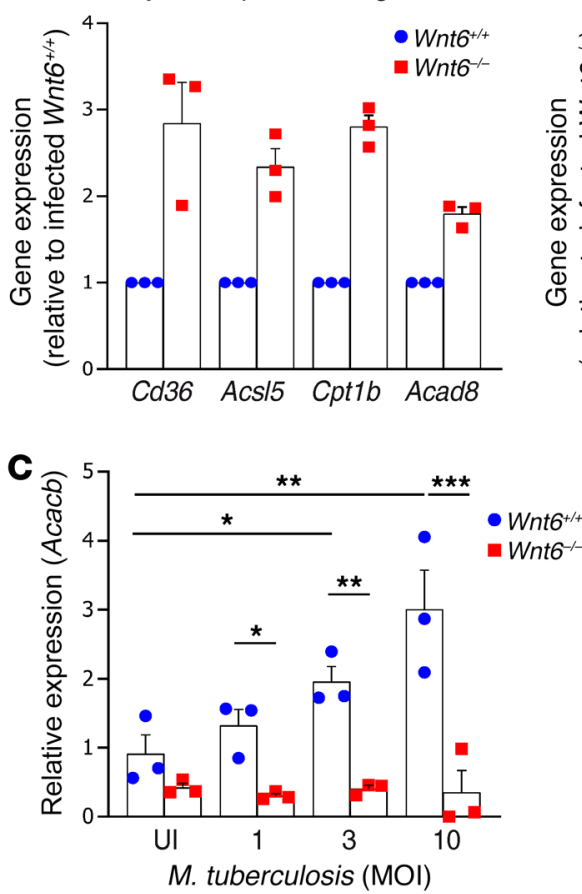

E

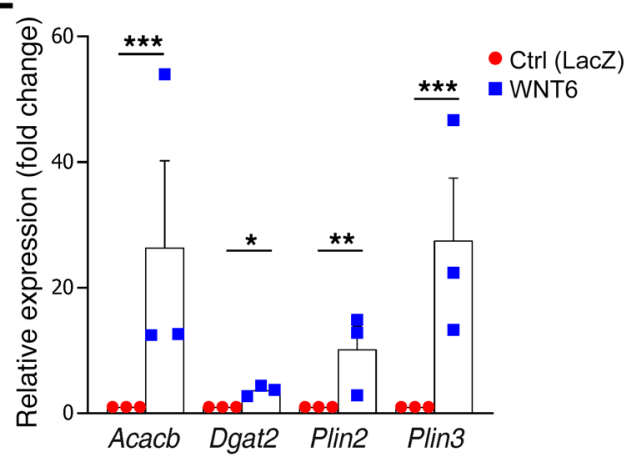

G

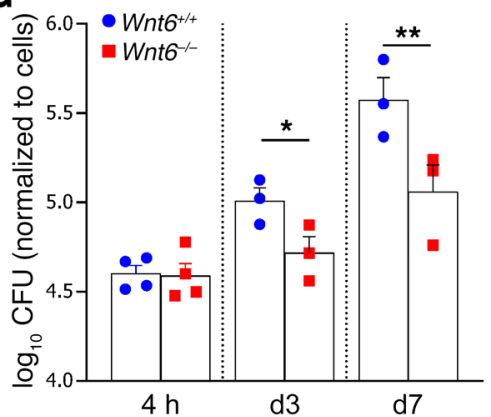

B

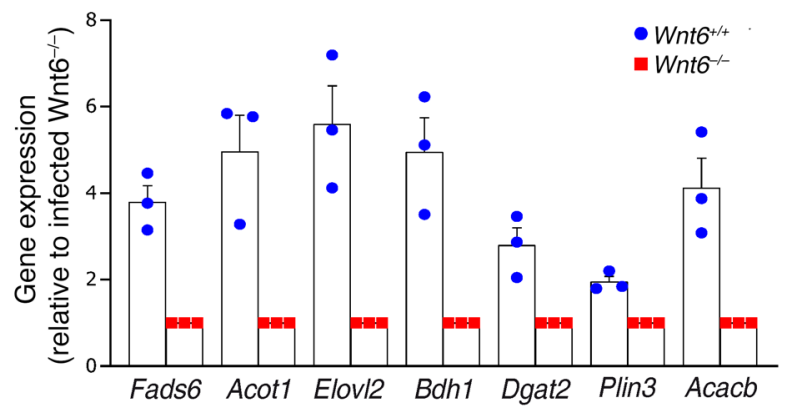

D

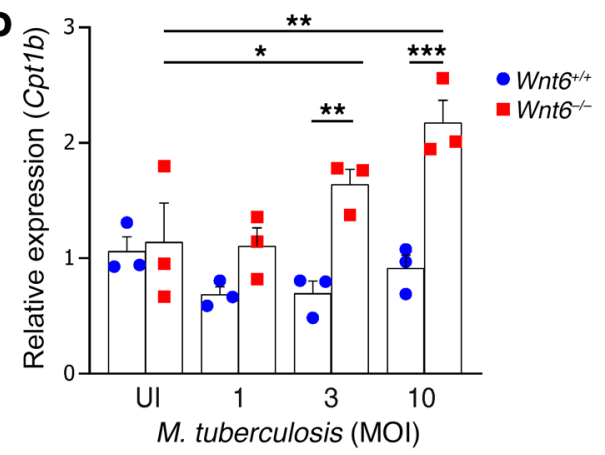

$\mathbf{F}$

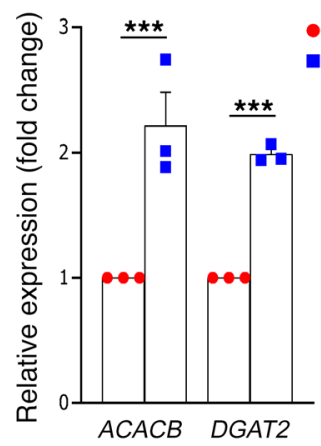

H

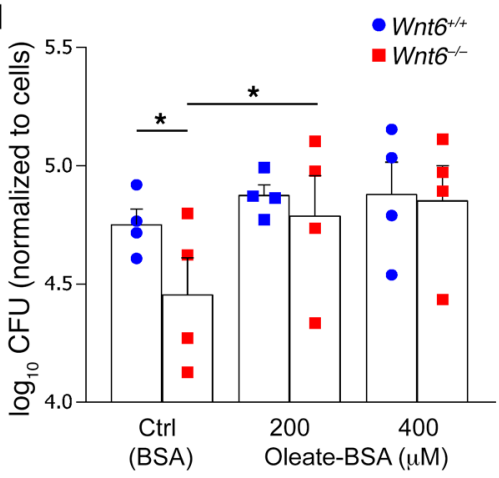

Figure 3. WNT6-mediated changes in host cell lipid metabolism promote Mtb survival in macrophages. (A and B) Microarray-based gene expression analysis of Mtb-infected (MOI 3:1, 24 hours) Wnt6 ${ }^{+/+}$and Wnt6-1- BMDMs. Fold expression of statistically significantly regulated genes associated with fatty acid uptake and degradation (A) or lipid synthesis and storage (B) is depicted; $n=3$. (C and D) RT-qPCR-based gene expression analysis of $\mathrm{Wnt6}^{+/+}$and Wnt6 $6^{-1-}$ BMDMs infected for 24 hours with various doses (MOls) of Mtb; $n$ = 3. (E) RT-qPCR-based gene expression analysis of WNT6-overexpressing (WNT6) or control [Ctrl (LacZ)] NIH 3T3 cells; $n=3$. (F) RT-qPCRbased gene expression analysis of hMDMs treated with WNT6 conditioned medium (WNT6 CM) or control conditioned medium (Ctrl CM) for 24 hours; $n=3$. (C) CFU analysis of Mtb-infected (MOI 1:1) Wnt6 ${ }^{+/+}$or Wnt6-/- BMDMs at days 0 (4 hours), 3, and 7 p.i.; $n=3-4$. (H) CFU analysis of Mtb-infected (MOI 0.1:1) Wnt6 W/+ $^{+/}$ or Wnt6 ${ }^{-/-}$BMDMs at day 7 p.i. after incubation with BSA (Ctrl) or various concentrations of oleate$\mathrm{BSA} ; n=4$. Statistical analyses were carried out using 1-way ANOVA with Holm-Šidák's multiple-comparison test except for microarray-based gene expression analysis (A and $\mathbf{B}$ ), which was conducted as described in Methods. ${ }^{*} P \leq 0.05$, ${ }^{*} P \leq 0.01$, ${ }^{* * *} P \leq 0.001$. All data are depicted as mean \pm SEM. factor (Supplemental Figure 4D). Moreover, acidification rates of Mtb-containing compartments were similar between $\mathrm{Wnt}^{+/++}$and Wnt $6^{-/-}$macrophages as determined by fluorescence microscopy analyses of the intracellular localization of GFP-Mtb (green) and LysoTracker dye (red) (Supplemental Figure 4E).

In order to test whether a reduced availability of lipid substrates is the cause of the impaired survival of Mtb in $W n t 6^{-/-}$cells, we determined Mtb CFUs in $W_{n t} 6^{+/+}$and $W n t 6^{-/-}$macrophages supplemented with fatty acids (oleate-BSA, Figure $3 \mathrm{H}$ ). In this set of experiments, macrophages were infected with a lower dose of Mtb (MOI 0.1:1), as we found that higher doses of Mtb in combination with fatty acids cause pronounced cell death, which confounds the CFU results. At day 7 p.i., CFU levels in control-treated (BSA-treated) cultures were reduced approximately 2-fold in Wnt $6^{-/-}$macrophages compared with $\mathrm{Wnt}^{+/+}$cells. Addition of $200 \mu \mathrm{M}$ oleate-BSA to $\mathrm{Wnt}^{-/-}$macrophages led to significantly enhanced CFU numbers, which were similar to those in $\mathrm{Wnt6}^{+/+}$ BMDMs. Higher oleate-BSA concentrations $(400 \mu \mathrm{M})$ also led to a 
A

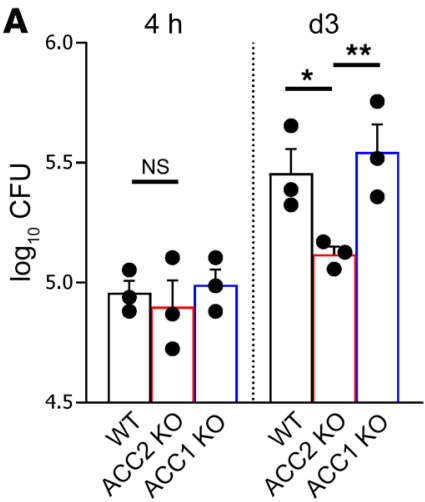

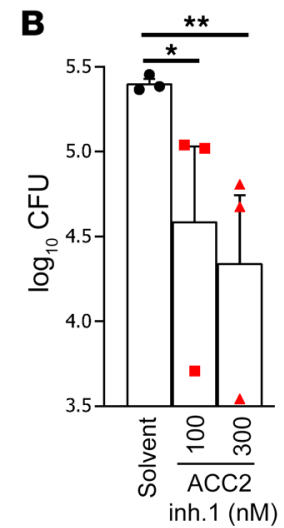
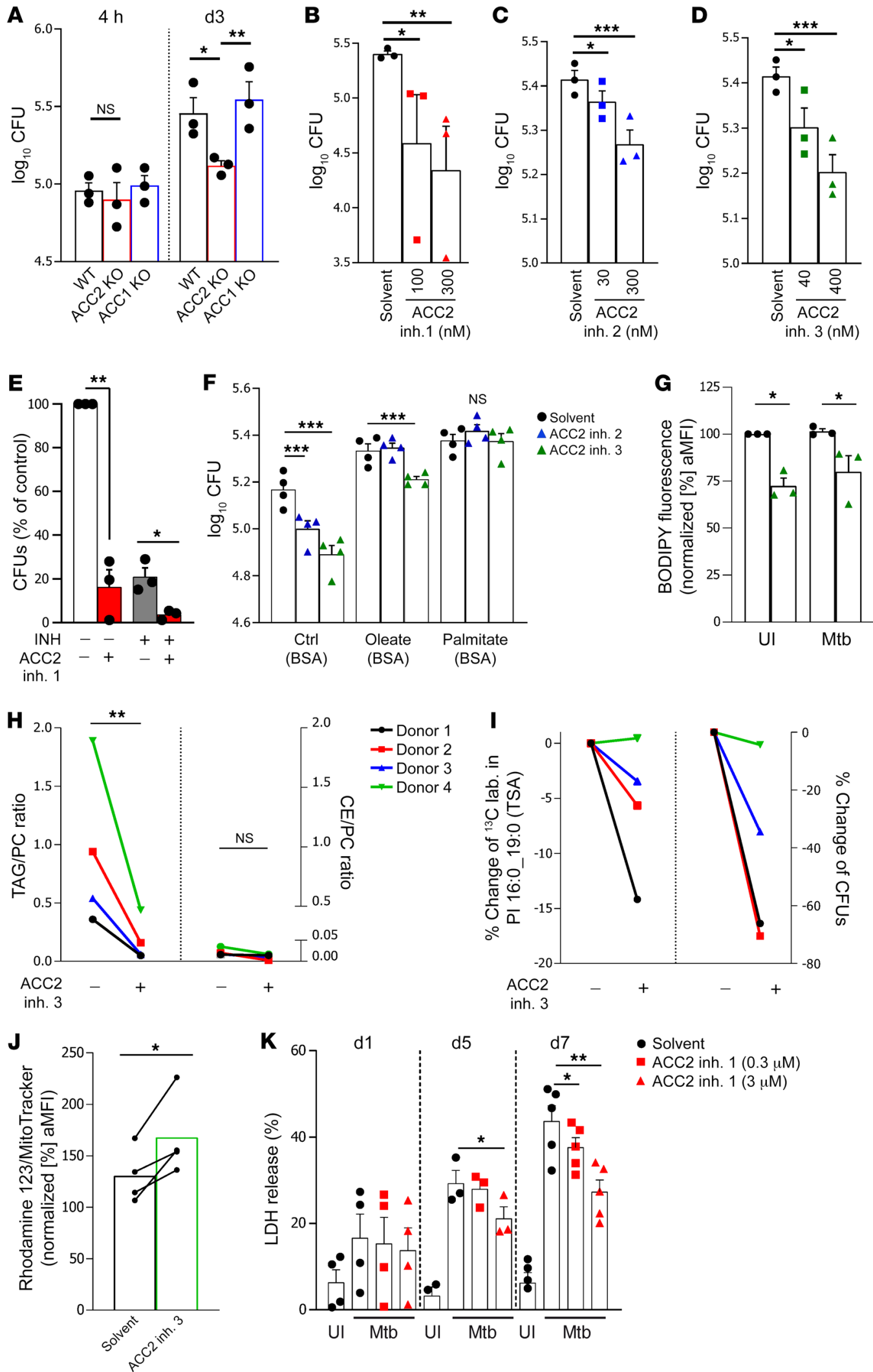
Figure 4. ACC2-dependent changes in host cell lipid metabolism promote Mtb survival in macrophages. (A) CFU analysis of Mtb-infected (MOI 0.5:1) wild-type (WT), ACC1-KO, and ACC2-KO human macrophage-like cells (BLaER1 macrophages) at days 0 (4 h) and 3 p.i. (d 3 ); $n=3$. (B-D) CFU analysis of Mtb-infected (MOI 1:1) hMDMs day $7 \mathrm{p}$.i. after incubation in the absence (solvent) and presence of ACC2 inhibitors; $n=3$. (E) In the same set of experiments depicted in B, cells were also treated with isoniazid (INH; $0.03 \mu \mathrm{g} / \mathrm{mL}$ ) or a combination of ACC2 inhibitor (300 nM) and INH. (F) CFU analysis of Mtb-infected (MOI 0.5:1) hMDMs day 7 p.i. after incubation with oleate-BSA or palmitate-BSA $(400 \mu \mathrm{M})$ and ACC2 inhibitors (2: $300 \mathrm{nM} ; 3: 400 \mathrm{nM}) ; n=4$. (G) Flow cytometry-based quantification of neutral lipids in uninfected (UI) and Mtb-infected (MOI 0.1:1) hMDMs at day 7 p.i.; $n=3$. (H and I) Prelabeled ( $200 \mu \mathrm{M}^{13} \mathrm{C}$-oleate-BSA) hMDMs were infected with Mtb (MOI 1:1) and incubated in the absence ("-", solvent) and presence of ACC2 inhibitor 3 ("+", $400 \mathrm{nM}$ ) for 7 days. Mass spectrometry-based quantification of relative TAG $(\mathbf{H}$, left) and CE (H, right) levels, as well as the change (relative to solvent, in percent) of isotope labeling in tuberculostearic acid (TSA; $C 19: 0,{ }^{12} \mathrm{C}_{1}{ }^{13} \mathrm{C}_{18}$ ) from the mycobacterial membrane lipid PI 16:0_19:0 (I, left). CFU values of the same samples (I, right); $n=4$. (J) Flow cytometry-based quantification at day 3 p.i. of the relative mitochondrial activity of Mtb-infected (MOI 0.1:1) hMDMs treated with solvent or ACC2 inhibitor $3(400 \mathrm{nM}) ; n=4$. (K) Lactate dehydrogenase (LDH) release from hMDM cultures at days 1,5 , and 7 p.i.; $n=3-5$. Statistical analyses were carried out using 1-way ANOVA with Holm-Šidák's multiple-comparison test; ${ }^{*} P \leq 0.05,{ }^{* *} P \leq 0.01,{ }^{* *} P \leq 0.001$.

comparable bacterial burden in $W n t 6^{+/+}$and $W n t 6^{-/-}$cells. Together, these data strongly suggest that WNT6 promotes Mtb survival in macrophages by regulating host cell lipid metabolism.

ACC 2 activity promotes $M t b$ survival in macrophages. To assess the role of the identified WNT6 target enzyme ACC2 during Mtb infection, we generated functional protein knockouts of ACC isoforms by CRISPR/Cas9-mediated genome editing in the human macrophage-like BLaER1 cell line $(35,36)$. CFU analyses of wild-type, ACC1-deficient, and ACC2-deficient cells 4 hours p.i. revealed a comparable uptake of Mtb by all cell types (Figure 4A). In contrast, at day 3 p.i., we observed 2.2-fold and 2.6-fold reduced Mtb CFUs in ACC2-deficient cells compared with wild-type and ACC1-deficient macrophages, respectively. Next, we treated primary human macrophages (hMDMs), in which Mtb bacterial numbers prominently increased over 7 days (Supplemental Figure $4 \mathrm{~F}$ ), with 3 structurally different pharmacological ACC2 inhibitors (Figure 4, B-D). All tested compounds reduced Mtb bacterial numbers on day 7 p.i. dose-dependently when compared with solvent control, albeit with varying efficacy (ranging from 1.4- to 6.5-fold CFU reduction). Targeting ACC2 as a host metabolic enzyme may conceivably complement pathogen-directed antibiotic treatments of TB. Therefore, we also tested the effect of ACC2 inhibition on Mtb CFUs in combination with the first line anti-TB drug isoniazid (INH), which was applied at suboptimal concentration $(0.03 \mu \mathrm{g} /$ $\mathrm{mL}$ ). ACC2 inhibitor 1 or INH alone led to a 6.5 -fold and a 4.7 -fold reduction of bacterial counts, respectively, when compared with solvent control (Figure 4E). Treating cells with a combination of both drugs resulted in a more than 20 -fold reduction of intracellular bacteria, revealing an almost additive effect of these drugs. Notably, the inhibitors tested did not exert toxic effects on human macrophages in the absence nor in the presence of INH (Supplemental Figure 5, A and B). Moreover, the inhibitor concentrations used did not inhibit Mtb growth in liquid culture as indicated by comparable fluorescence signals between control-treated (solvent-treated) and ACC2 inhibitor-treated GFP-expressing Mtb bacteria (Supplemental Figure 5C). Finally, we did not observe a direct effect of ACC2 inhibition on the immediate inflammatory response of hMDMs to Mtb as determined by measurement of TNF- $\alpha$ release (Supplemental Figure 5D).

In order to assess whether a reduced availability of lipid substrates is the cause of the reduced survival of Mtb in macrophages lacking ACC2 activity, we determined Mtb CFUs in ACC2 inhibitor-treated macrophages supplemented with fatty acids. In this set of experiments, we again decided to reduce the infectious dose in order to limit the influence of cell death on the resulting CFU data. Both oleate-BSA and palmitate-BSA increased the number of Mtb bacteria at day 7 p.i. when added to hMDM cultures (Supplemental Figure 5E). Exogenously added fatty acids - depending on the efficacy of the inhibitor and the type of fatty acid used - increased the number of intracellular Mtb bacteria to levels that were indistinguishable between ACC2 inhibitor-treated and solvent-treated cells (Figure 4F). Together, our studies on cells lacking either WNT6 or active ACC2 reveal that WNT6/ACC2-mediated lipid metabolic changes are causative for the Mtb survival-promoting effect of this signaling pathway in macrophages.

ACC2 inhibition lowers TAG levels in infected macrophages and utilization of host cell fatty acids by Mtb. Next, we examined the impact of ACC2 inhibition on neutral lipid levels in macrophages in the absence and presence of Mtb. Flow cytometry-based quantification of BODIPY 493/503 signals showed that ACC2 inhibition to a similar extent decreased neutral lipid levels in uninfected and infected hMDMs on day 7 p.i. (Figure 4G). This reveals an infection-independent effect of ACC2 activity on neutral lipid storage in macrophages. Mtb infection itself did not increase BODIPY signals in hMDMs at day 7 p.i.

Neutral lipids are a known source of lipid nutrients for Mtb (37, 38). Among these Mtb utilizes oleic acid (C18:1) to synthesize, e.g., tuberculostearic acid (TSA) (C19:0) by attaching a methyl group (C1) (39), which allows Mtb to form the mycobacterial plasma membrane lipid phosphatidylinositol (PI) 16:0_19:0 (TSA) (40). In contrast to TAG inclusions within Mtb, which are formed, e.g., in response to hypoxia (37), PI 16:0_19:0 (TSA) formation is directly linked to bacterial replication (40). We hypothesized that Mtb exploits WNT6/ACC2-mediated storage of TAG-rich lipid droplets in macrophages by utilizing them as a carbon source. We therefore assessed whether ACC2 inhibition in macrophages influences the utilization of host-derived oleic acid by Mtb. We preincubated hMDMs with isotopically labeled ${ }^{13} \mathrm{C}$-oleic acid (oleate-BSA). Subsequently, cells were infected with Mtb and incubated in the absence and presence of ACC2 inhibitor. Mass spectrometric analysis revealed that ${ }^{13} \mathrm{C}$-oleate was effectively incorporated into TAG, CE, and PC species of the host cell (Supplemental Figure 6 and Supplemental Table 1). ACC2 inhibitor treatment reduced TAG concentration at day 7 p.i. in all donors, while we observed no effect of ACC2 inhibition on CE levels (Figure 4H). Notably, CEs were more than a magnitude less abundant compared with TAGs. Next, we assessed whether Mtb utilized less host-derived oleic acid $\left({ }^{13} \mathrm{C}_{18}\right.$-OA) to form PI 16:0_19:0 $\left({ }^{12} \mathrm{C}_{1}{ }^{-13} \mathrm{C}_{18}\right.$ TSA) (Supplemental Figure 7) when ACC2 inhibitor was present (Figure 4I, left, and Supplemental Figure 5H). Donors 1, 2, and 3 showed a reduction of 
A

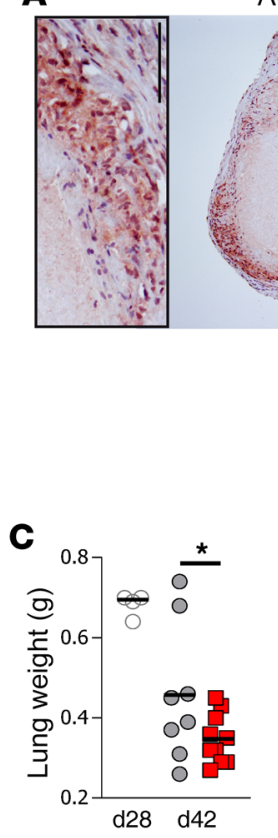

ACC2

B

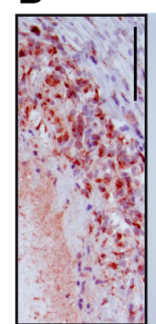

CD68

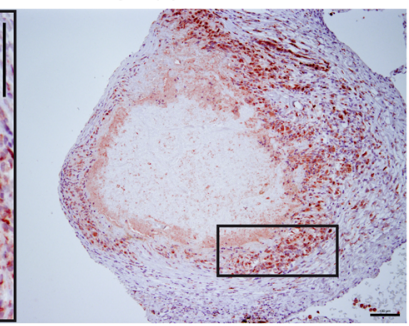

Oral gavage (twice a day)

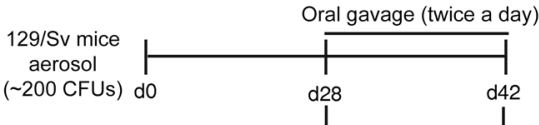

$\stackrel{\text { d28 }}{\square \text { Pretreatment Isoniazid }}$

Isoniazid + ACC2 inh. 3
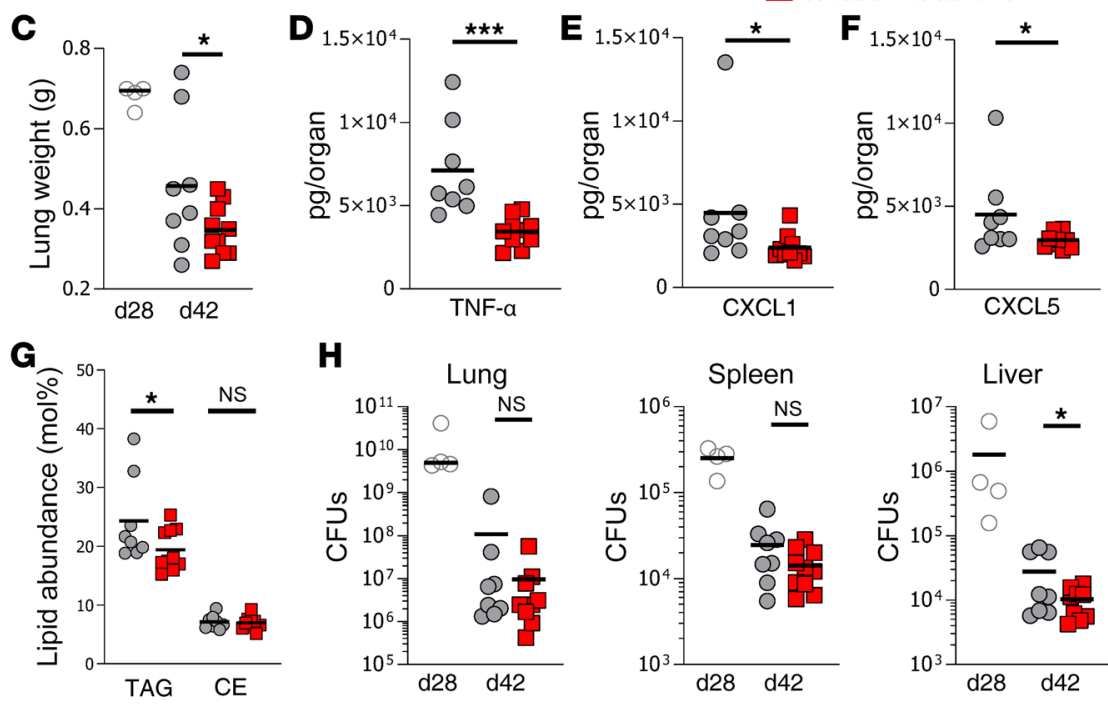

H
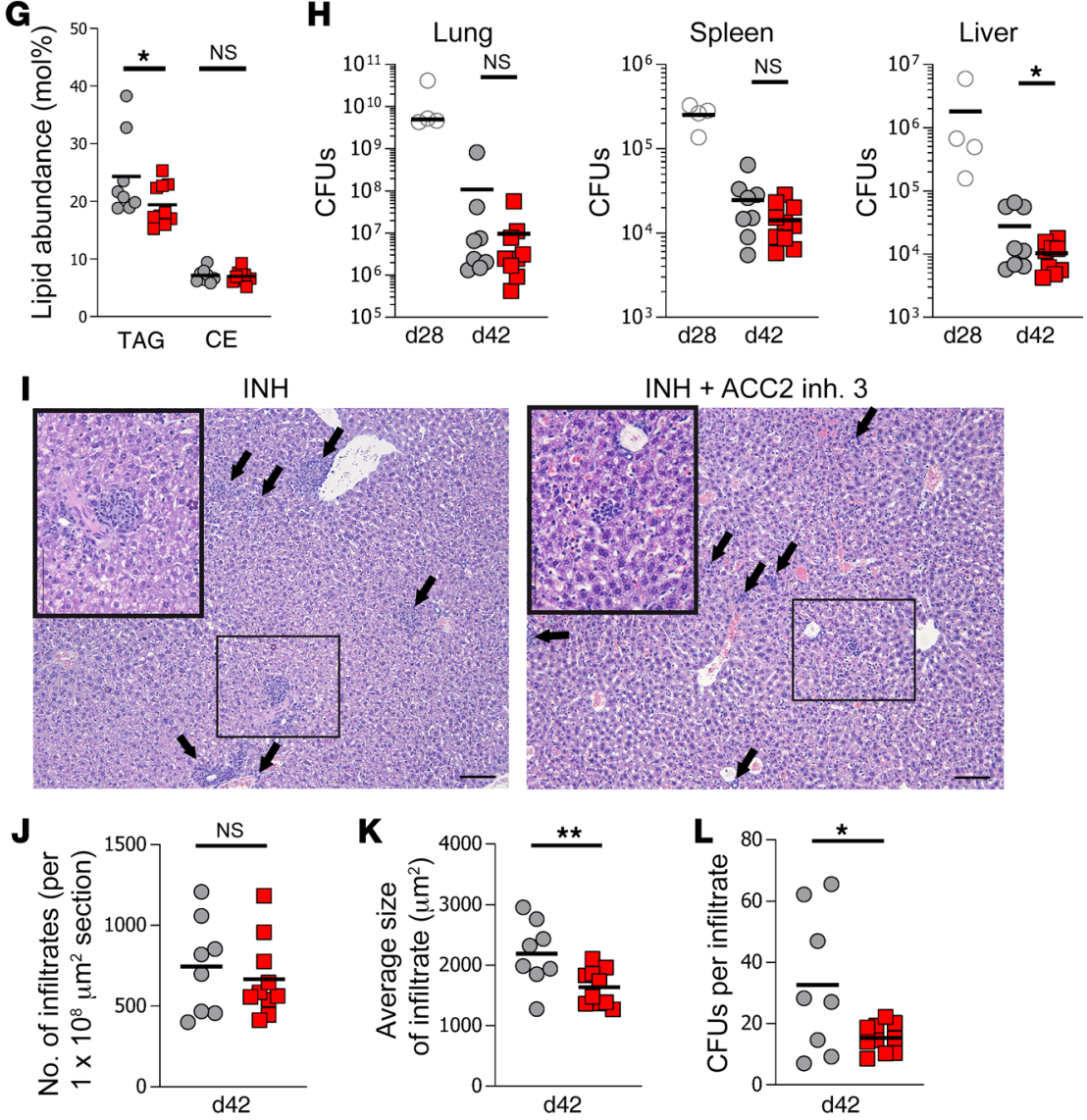

Figure 5. The role of ACC2 during Mtb infection in vivo. (A and B) Immunohistochemical stainings for ACC2 (A) and the macrophage/monocyte marker CD68 (B) of consecutive lung tissue sections derived from a TB patient. A representative observation from 2 independent observations is shown. Results of these stainings are again shown in Supplemental Figure 8A. (C-L) In vivo effect of adjuvant ACC2 inhibitor treatment on Mtb infection in TB-susceptible mice. After 28 days of infection with Mtb ( 200 CFUs), 129/ Sv mice either were left untreated (pretreatment, $d$ 28 p.i., $n=4$, white symbols) or were treated for 14 days with INH alone ( $10 \mathrm{mg} / \mathrm{kg}$ body weight, $n=8$, gray symbols) or with ACC2 inhibitor 3 (ND-646, 25 $\mathrm{mg} / \mathrm{kg}$ body weight) plus INH ( $n=10$, red symbols). Lung weights (C), lung cytokine and chemokine levels (D-F), TAG and CE abundance in the lung (G), and mycobacterial loads in lung, spleen, and liver $(\mathbf{H})$ were determined. (I-K) Mononuclear infiltrates in the livers of Mtb-infected mice (I, black arrows), and quantification of infiltrate numbers (J), infiltrate size (K), and the average CFUs per infiltrate (L). Statistical analyses were carried out using a 1-tailed, unpaired Student's $t$ test; ${ }^{*} P \leq 0.05,{ }^{* *} P \leq 0.01,{ }^{* * *} P \leq 0.001$. Data are depicted as scatter dot plots with line at mean. Scale bars: $100 \mu \mathrm{m}$.
${ }^{13} \mathrm{C}$-label incorporation into PI 16:0_19:0, which was accompanied by a decrease in Mtb bacterial numbers (Figure 4I, right). Both parameters remained almost unchanged in samples from donor 4, which showed up to 8-fold increased TAG levels after ACC2 inhibition when compared with samples from donors 1, 2, and 3 (TAG/ PC ratio: 0.34 to $0.04,0.11$, and 0.04 , respectively). We conclude from these data that Mtb utilizes less host-derived oleic acid to synthesize bacterial membrane lipids when TAG levels in macrophages are sufficiently reduced by ACC2 inhibition. This finding supports the notion that Mtb exploits WNT6/ACC2-mediated storage of TAGs in macrophages to gain access to lipid nutrients.

ACC2 inhibition enhances mitochondrial activity and limits Mtb-induced cell death of macrophages. ACC2 activity is known to impair mitochondrial fatty acid oxidation through CPT1 inhibi- 
tion $(33,34,41)$. Consistent with that, we observed that mitochondria were metabolically more active in ACC2 inhibitor-treated hMDMs as well as in ACC2-KO BlaER1 macrophages compared with respective control cells as indicated by increased relative fluorescence signals of the membrane potential-sensitive fluorochrome rhodamine 123 (42) (Figure 4J and Supplemental Figure $5 \mathrm{~F}$ ). Based on these data and the previous observation that enhanced fatty acid oxidation upon modulation of the ACC/CPT1 pathway is part of a protective mechanism against fatty acid-induced cell death (43), we proceeded to determine the viability of Mtb-infected macrophages in the absence or presence of ACC2 inhibitors. Thus, we measured the release of lactate dehydrogenase (LDH) as a marker of cell membrane disruption (44). Macrophages infected with Mtb showed a marked increase in LDH release during the course of infection in a time-dependent manner with an approximately $43 \%$ maximum release at day 7 p.i. (Figure $4 \mathrm{~K})$. Strikingly, when treating infected cells with ACC2 inhibitor we observed a statistically significant reduction of $\mathrm{LDH}$ release in a dose-dependent manner in comparison with solvent control (up to $~ 50 \%$ reduction of $\mathrm{LDH}$ release). In contrast, we observed no effect of ACC2 inhibitor treatment on LDH release when cells remained uninfected (Supplemental Figure 5G). In summary, these findings suggest that ACC2 inhibition increases fatty acid oxidation in mitochondria during infection and reduces Mtbinduced cell death of macrophages.

The expression and function of ACC2 during Mtb infection in vivo. To elucidate the role of ACC2 during Mtb infection in vivo, we stained lung tissue sections from a TB patient with different ACC antibodies (Supplemental Figure 8A). This analysis revealed a strong signal for ACC2 in the periphery of necrotizing granulomas coinciding with the presence of $\mathrm{CD} 68^{+}$cells (boxes, Figure 5, $\mathrm{A}$ and $\mathrm{B})$, suggesting that macrophage ACC2 plays a role during active TB in humans. Next, we aimed to investigate the functional role of ACC2 in vivo, using an animal model of Mtb infection, and thus stained for both ACC isoforms in the lungs of Mtb-infected mice. We observed only a weak staining for ACCs in C57BL/6 mice although they were infected with a high dose ( 1000 CFUs) of Mtb (Supplemental Figure 8B). In contrast, numerous ACCpositive cells were easily detectable in low-dose-infected 129/ Sv mice (Supplemental Figure 8C), which are known to develop a TB-susceptible phenotype resembling primary progressive TB disease in humans (45). Consequently, we chose 129/Sv mice that were infected with Mtb for 28 days and treated with ACC2 inhibitor 3 by oral gavage. ACC2 inhibitor 3 (ND-646) was selected for our in vivo study because of the advanced preclinical (46) and clinical status of this group of compounds. ND-646 represents a structurally closely related derivative of ND-630, which has been successfully used in a phase II clinical trial with patients suffering from nonalcoholic fatty liver disease $(47,48)$.

Seven days after the start of treatment with the ACC2 inhibitor, no substantial changes with regard to Mtb bacterial burden were observed when homogenates of lung, liver, and spleen of ACC2 inhibitor-treated mice were compared with those of vehicle control-treated mice (Supplemental Figure 8D). Since targeting of host ACC2 would always be an adjunct to pathogen-directed TB therapy, we also combined ACC2 inhibitor with INH in vivo. Two weeks of treatment with ACC2 inhibitor combined with INH significantly reduced lung weights of infected mice by $25 \%$ when compared with INH alone (Figure 5C). Moreover, cytokine quantification in lung samples revealed a reduced formation of the proinflammatory cytokine TNF- $\alpha$ (about 2 -fold) as well as slightly reduced levels of the chemoattractants CXCL1 and CXCL5 in mice treated with INH plus ACC2 inhibitor compared with INH-treated animals (Figure 5, D-F). These findings might indicate a reduced presence of immune cells in the lungs when ACC2 is inhibited; however, we did not find significant differences with regard to the lung histopathology between the treatment groups (Supplemental Figure 8E). Mass spectrometric analyses showed no change in the abundance of $\mathrm{CE}$ in the lungs when comparing treatment groups (Figure $5 G$ ). In contrast, we found a significantly reduced TAG abundance in the lungs of mice treated with ACC2 inhibitor and INH, indicating a specific reduction ( $20 \%)$ of TAG levels by ACC2 inhibition also in Mtb-infected mice in vivo. Under these conditions, Mtb CFUs in the lungs and spleens of infected mice were comparable between both treatment groups (Figure $5 \mathrm{H}$ ). In contrast, we found a significant (2.7-fold) reduction in Mtb CFUs in the livers of mice treated with INH plus ACC2 inhibitor compared with INH-treated animals. Detailed histological analyses identified that decreased CFU numbers were accompanied by a reduced liver pathology in these animals (Figure 5I). We observed a similar number of mononuclear cell infiltrates in the livers of mice treated with INH versus INH plus ACC2 inhibitor (Figure 5J), suggesting similar dissemination of Mtb into the liver from the lung. However, the average size of these infiltrates was significantly reduced by more than $30 \%$ (Figure $5 \mathrm{~K}$ and Supplemental Figure 9), indicative of reduced Mtb replication within these granulomatous foci. Indeed, our data reveal an average reduction of Mtb bacterial burden per liver infiltrate of about 50\% (Figure 5L). These findings from the livers suggest that ACC2 activity contributes to Mtb replication and disease-induced pathology also in vivo.

\section{Discussion}

Foamy macrophages, abundantly found within granulomas of TB patients, provide a nutrient-rich host cell environment for Mtb and are involved in tissue pathology during disease (14). However, the detailed mechanisms by which infection induces the development of these lipid-laden cells are still unclear. Our study uncovers that the WNT ligand WNT6 acts as a foamy macrophage-promoting factor during pulmonary TB. We found prominent WNT6 expression in cells showing characteristics of foamy macrophages in the lungs of Mtb-infected mice and TB patients. Mechanistically, we found that WNT6-induced ACC2 activity in macrophages mediates a metabolic shift away from oxidation of fatty acids toward intracellular storage into TAGs. Mtb exploits WNT6/ACC2dependent metabolic changes in macrophages as it gains access to TAG-derived lipid nutrients, which facilitate its survival within the host cell (summarized in the graphical abstract).

The WNT signaling pathway is critically involved in the regulation of energy homeostasis. Notably, its ligands act on cellular or tissue metabolism in a cell type- and microenvironment-dependent manner. This is exemplified by WNT3a, which promotes the accumulation of neutral lipids in epithelial cells (49), while reducing the synthesis of neutral lipids in hepatocytes (50). Another example is WNT5a, which increases fatty acid oxidation in den- 
dritic cells in the context of melanoma, leading to reduced inflammatory and antitumor responses as part of a metabolic reprogramming of cells (51). In contrast, WNT5a in adipose tissue promotes inflammation and contributes to obesity-associated metabolic dysfunction (52). In the context of Mtb infection, WNT5a exerts proinflammatory functions, while WNT3a and WNT6 dampen inflammatory responses of macrophages (19). Our study, to the best of our knowledge, is the first that reveals a regulatory function of a WNT ligand on macrophage metabolism during Mtb infection. We show that WNT6 drives neutral lipid accumulation in macrophages in addition to its previously uncovered antiinflammatory properties (20). Notably, WNT6 is also upregulated during inflammatory bowel disease and allergic asthma $(53,54)$. Thus, the current findings describing a newly identified function of WNT6 in macrophages can help to understand the pathogenesis of other chronic inflammatory diseases.

Pathogens can trigger foam cell formation in a Toll-like receptor-dependent (TLR-dependent) manner (55). From a metabolic perspective, exposure of macrophages to already one single TLR ligand increases TAG storage $(12,56)$, enhances fatty acid uptake $(12,57)$, and diminishes mitochondrial fatty acid oxidation even in the presence of sufficient oxygen $(12,57)$. We have previously shown that conserved bacterial structures and various mycobacterial species, including Mtb, induce WNT6 expression in macrophages via TLR/NF- $\mathrm{B}$ signaling (20). We now show that WNT6 signaling induces the expression of key lipid metabolic enzymes including DGAT2 and ACC2. The latter is a regulatory enzyme that is well known to increase neutral lipid storage in cells by blocking CPT1 activity and thereby preventing oxidation of fatty acids in mitochondria $(33,34,41)$. Indeed, we found that WNT6/ ACC2 signaling limits mitochondrial activity of macrophages, leading to a shift in lipid metabolism away from oxidation of fatty acids toward TAG synthesis and their storage in lipid droplets. During chronic Mtb infection, mycobacterial TLR ligands lead to recurring activation of macrophages. Thus, it seems likely that the mycobacteria-mediated and TLR-dependent differentiation of macrophages into a foamy phenotype is in part dependent on WNT6/ACC2-induced changes in cellular fatty acid metabolism.

Known mechanisms by which Mtb interferes with cellular lipid homeostasis include epigenetic alterations (23) or activation of cells with keto-mycolic acid $(15,25)$. Studies on the nuclear receptor TR4, the antilipolytic GPR109A, and miRNA-33 could relate impaired survival of Mtb in macrophages to reduced neutral lipid levels $(24,25,58)$. However, in these studies it has not been elucidated how bacteria benefit from neutral lipid storage in host cells. Our study uncovers that WNT6/ACC2 signaling drives TAG storage in macrophages. Furthermore, Mtb CFUs were reduced in macrophages lacking functional WNT6 or ACC2, strongly suggesting that WNT6/ACC2 signaling promotes survival or growth of Mtb inside its main host cell. Although we cannot exclude that variations in bacterial clumping contribute to the observed CFU changes, we propose that impaired survival of Mtb in the absence of functional WNT6 and ACC2 is the most likely explanation. Consistent with that, we found that ACC2 inhibition limits the incorporation of host-derived oleic acid into phospholipids of Mtb, which are needed for bacterial cell membrane synthesis and replication. These results on the role of the WNT6/ACC2 pathway during Mtb infection suggest that intracellular bacteria require sufficient access to TAG-derived fatty acids to survive or even replicate within host cells. It has previously been shown that the intracellular survival of Mtb can be dependent on the access to fatty acids from oleic acid-induced macrophage lipid droplets (38). Moreover, Mtb proteins involved in lipid uptake into the bacteria are required for full bacterial virulence in vivo $(59,60)$, which supports the notion that a reduction in the availability of fatty acids from the host could affect the survival or replication of Mtb in macrophages. On the other hand, it has also been shown that - e.g., when macrophages face hypoxic conditions - host-derived fatty acids are converted and stored by Mtb into TAG inclusions in the bacteria, which do not fuel bacterial replication under these conditions (37). Ultimately, the microenvironment of Mtb and its host cell - in particular, local oxygen levels - is decisive for whether Mtb actively replicates or acquires a dormancy-like phenotype. We are well aware that the molecular consequences of inhibition of the ACC2 signaling cascade for macrophage-Mtb interaction have not been fully elucidated here. This would require in-depth analyses of, e.g., trafficking of fatty acids within the host as well as their transport into Mtb and its membrane, which are beyond the scope of the current study.

Lipid droplets are multifunctional organelles, comprising a core of neutral lipids (e.g., TAG and CEs) and a surrounding phospholipid monolayer shell that harbors a variety of different proteins and enzymes (61). Mtb is not able to acquire host lipids from IFN- $\gamma$-induced lipid droplets rich in CE (62), while we observed in our study that Mtb can utilize host fatty acids from TAGs. Bacterial and host-derived signals may induce the formation of differentially composed subsets of lipid droplets rich in either TAG or CE. Thus, the amount of Mtb bacteria in relation to the extent of the host response may define whether TAG- or CE-rich lipid droplets are formed. Depending on their composition, lipid droplets could either contribute to host defense, e.g., by acting as a platform for the synthesis of small lipid mediators (62), or promote bacterial survival by being exploited by Mtb as a carbon source.

While more than 10 drugs are currently available for TB treatment, the treatment success of multidrug-resistant and extensively drug-resistant Mtb strains is as low as $50 \%$ on a global level. This stimulated intensive research to develop new anti-TB drugs, but also to explore alternative treatment concepts including hostdirected therapies (HDTs), which bear the promise of enhancing the efficacy of classical TB drugs and preventing resistance development (2). The findings of our study suggest that inhibiting TAG storage in macrophages could deprive Mtb of essential lipid nutrients needed for its intracellular survival and growth, which would represent a novel HDT approach that could complement and improve antibiotic-based TB treatment. Our in vivo data from an experimental murine TB infection model show a significant effect of adjuvant ACC2 inhibitor treatment on Mtb replication in the livers of TB-susceptible mice, which was accompanied by a reduced liver pathology. We conclude from these findings that under distinct conditions ACC2 represents a host factor that promotes mycobacterial replication also in vivo. However, to the best of our knowledge, the liver does not play a critical role during pulmonary TB development in humans (63), and liver-restricted manifestations of extrapulmonary TB are very rare (64). Adjuvant ACC2 
inhibitor treatment did not impact Mtb bacterial numbers in the lung, representing the key organ during TB infection, although we found reduced lung weights, TAG levels, and proinflammatory cytokine production. This may indicate that ACC2 inhibitor accumulated to higher levels in the liver, an organ in which a firstpass effect has been amply documented (65). Further in-depth studies to determine the optimal route, dosage, and regimen for administration of ACC2 inhibitors in combination with antibiotics are needed to determine the conditions that would result in a reduced bacterial load in the lungs and thereby improve treatment outcomes. Targeting of cellular fatty acid metabolism using ACC inhibitors has been successfully employed in patients suffering from hepatic steatosis (33), but also in preclinical models of nonsmall cell lung cancer (46). Thus, in a long-term perspective, targeting of host metabolic enzymes such as ACC2, which results in a limited availability of host lipids as a carbon source for Mtb, may represent a promising approach for an adjunct TB therapy.

\section{Methods}

Mice and macrophages. 129S2/SvPasOrlRj and C57BL/6N mice were purchased from Janvier and from Charles River Laboratories (Sulzfeld, Germany), respectively. NMRI $W n t 6^{+/+}, W n t 6^{-/}$, and $I L-13$-overexpressing mice, generated (66) and provided by Andrew McKenzie (MRC Laboratory of Molecular Biology, Cambridge, United Kingdom), were raised and maintained under specific pathogen-free conditions. NMRI mice were originally obtained from Charles River Laboratories, and the Wnt6-null allele was generated as described previously (67). NMRI Wnt $^{-/-}$mice were generated by heterozygous mating at the Research Center Borstel.

To generate bone marrow-derived macrophages (BMDMs), bone marrow cells of mice were differentiated as described previously (68). To obtain quiescent tissue macrophages, peritoneal exudate cells were isolated from the resting peritoneal cavity of mice as described previously (69). Further details are provided in Supplemental Methods. To generate human monocyte-derived macrophages (hMDMs), peripheral blood monocytes (purity consistently $>92 \%$ ) were obtained by counterflow centrifugation from peripheral blood mononuclear cells of healthy blood donors. Subsequently, isolated cells were incubated for 7 days in Teflon bags (VueLife 72C, CellGenix) in VLE RPMI 1640 (Merck) containing 4\% human AB serum, 4 mM glutamine, 1\% penicillin/streptomycin (Merck), and $10 \mathrm{ng} / \mathrm{mL}$ recombinant human $\mathrm{M}$-CSF (Bio-Techne) as described previously (70). Cells were incubated in cell culture medium (VLE RPMI 1640, 10\% FCS (Pan-Biotech), 4 mM glutamine) with M-CSF omitted for further analyses.

Mtb strains and in vitro growth assays. Mtb strain H37Rv (ATCC 27294, American Type Culture Collection), GFP-expressing Mtb (H37Rv:pMN:437 [ref. 71] or H37Rv:psVM4 [ref. 72]), and mCherryexpressing Mtb (73) were harvested at mid-log phase $\left(\mathrm{OD}_{600 \mathrm{~nm}} \sim 0.3\right)$ and stored as frozen aliquots at $-80^{\circ} \mathrm{C}$ as described previously (70). For Mtb growth analysis in liquid culture, frozen aliquots were thawed and centrifuged $(2300 \mathrm{~g}, 10$ minutes), and bacteria in 7H9 medium supplemented with $10 \%$ Oleic Albumin Dextrose Catalase (OADC) (MilliporeSigma) were thoroughly homogenized by use of a syringe and a 26-gauge syringe needle. Growth of GFP- or mCherry-expressing Mtb in black, clear-bottom plates (MilliporeSigma) was estimated by measurement of fluorescence in a microplate reader (Synergy 2, BioTek Instruments).
Infection of macrophages and mice. For in vitro infection experiments, homogenized Mtb bacteria resuspended in cell culture medium were used to infect cells with the indicated dose of bacteria (MOI) for 4 hours $\left(37^{\circ} \mathrm{C}, 5 \% \mathrm{CO}_{2}\right)$, followed by extensive washing with HBSS (MilliporeSigma) to remove extracellular bacteria. Subsequently, cells were treated with solvent/carrier control, the indicated inhibitor, or fatty acids for up to 7 days $\left(37^{\circ} \mathrm{C}, 5 \% \mathrm{CO}_{2}\right)$.

Mice (female, aged 8-12 weeks) infected via the aerosol route with Mtb H37Rv $(21,74)$ were kept under barrier conditions and sacrificed at the indicated time point, and the organs were prepared and analyzed as previously described $(21,74)$.

For quantification of viable colony-forming units (CFUs), samples derived from homogenized tissue or cells (lysed by incubation with $2 \%$ saponin/HBSS) were serially diluted in $0.05 \%$ Tween- $80 / \mathrm{dH}_{2} \mathrm{O}$ and plated on $7 \mathrm{H} 10$ agar plates containing $10 \%$ heat-inactivated bovine serum (Merck). Plates were incubated for $3-4$ weeks at $37^{\circ} \mathrm{C}$. Before lysing of BMDMs, images were taken at defined positions of each well by use of a bright-field microscope (DM LB, Leica Biosystems) and a digital camera (Sight DS-L11, Nikon). The number of cells within a well was enumerated by analysis of images with a counting tool (Adobe Photoshop CS5 software, version 12.04 and earlier).

Stimuli and inhibitors. For in vitro infection experiments, DMSO (MilliporeSigma) was used to solubilize $N$-(1-(2'-(4-isopropoxyphenoxy)-2,5'-bithiazol-5-yl)ethyl)acetamide ("ACC2 inhibitor 1"; ab142090, Abcam), 5-[1'-(1-cyclopropyl-4-methoxy-3-methylindole6-carbonyl)-4-oxospiro[3H-chromene-2,4' -piperidine]-6-yl]pyridine-3-carboxylic acid ("ACC2inhibitor2," known as MK-4074 [ref.33]; MedChemExpress), and 1,4-dihydro-1-[(2R)-2-(2-methoxyphenyl)2-[(tetrahydro-2H-pyran-4-yl)oxy] ethyl]-a,a,5-trimethyl-6-(2-oxazolyl)-2,4-dioxothieno[2,3-d]pyrimidine-3(2H)-acetamide ("ACC2 inhibitor 3," known as ND-646 [ref. 46]; MedChemExpress). DMSO served as a solvent control ( $0.1 \%$ in cell culture medium).

${ }^{12} \mathrm{C}$-Oleic acid (pure, pharma grade; Applichem) and ${ }^{12} \mathrm{C}$-palmitic acid (MilliporeSigma) were conjugated to the carrier protein bovine serum albumin (BSA; Applichem or MilliporeSigma [low endotoxin, fatty acid free]). In brief, a solution of $20 \mathrm{mM}$ fatty acid in $0.01 \mathrm{M}$ $\mathrm{NaOH}$ was incubated at $70^{\circ} \mathrm{C}$ for 30 minutes, followed by dropwise addition of $1 \mathrm{M} \mathrm{NaOH}$ facilitating the solubilization of the fatty acid. Solubilized fatty acids were complexed to BSA in PBS at an 8:1 fatty acid/BSA molar ratio. The complexed fatty acids or BSA alone were added to serum-containing cell culture medium to achieve different fatty acid concentrations or a suitable control. Inhibitors and fatty acids were added to the cells after removal of extracellular bacteria by washing in order to avoid interference with bacterial uptake.

ACC2 inhibitor treatment of mice. To study the effect of ACC2 inhibition on Mtb infection in vivo, ACC inhibitor 3 (ND-646) (46) was administered to $129 / \mathrm{Sv}$ mice by oral gavage twice a day at a concentration of $25 \mathrm{mg} / \mathrm{kg}$ body weight. The corresponding volume of a vehicle solution $(0.9 \% \mathrm{NaCl}, 1 \%$ [vol/vol] Tween-80, 30\% [wt/vol] Captisol; CyDex Pharmaceuticals) with ND-646 omitted served as treatment control. Moreover, mice were treated either with isoniazid alone (10 $\mathrm{mg} / \mathrm{kg}$ body weight; MilliporeSigma) or with a combination of isoniazid with ACC2 inhibitor. Treatment was started at day 28 p.i. and conducted for a period of 7 days (vehicle vs. ACC2 inhibitor) or for 14 days with isoniazid and isoniazid plus ACC2 inhibitor.

NIH 3 T3 cells and generation of WNT6 conditioned medium. Wnt6-transfected NIH 3T3 cells were a gift from S. Vainio (University 
of Oulu, Oulu, Finland). In order to yield highly pure WNT6-expressing clones, single cells were placed in 96-well plates using a FACSAria IIu cell sorter (Becton Dickinson) with an automated cell deposition unit. The resulting clones were screened for Wnt6 mRNA expression and selected accordingly. Control-transfected (LacZ) NIH 3T3 cells were a gift from R. Kemler (Max Planck Institute of Immunobiology and Epigenetics, Freiburg, Germany). To generate conditioned medium (CM), culture supernatants of NIH 3T3 cells grown for 3 days were collected, filtered through a $0.2 \mu \mathrm{m}$ filter, and stored at $-80^{\circ} \mathrm{C}$ until further usage. $\mathrm{CM}$ derived from cells overexpressing and secreting WNT6 (referred to as WNT6 CM) or from a similar number of control (LacZ) cells (referred to as control CM) were used for stimulation experiments with macrophages.

BLaER1 cells and generation of functional protein knockouts using CRISPR/Cas9. B cell leukemia C/EBP $\alpha$ ER clone 1 (BLaER1) cells (35) were a gift from Thomas Graf (Center for Genomic Regulation, Barcelona, Spain) and were cultivated in VLE RPMI containing 10\% heat-inactivated FCS, $4 \mathrm{mM}$ glutamine, and 1\% penicillin/streptomycin. Functional protein knockouts of ACC1 and ACC 2 were generated by CRISPR/Cas9-mediated genome editing using the following guide RNA sequences for the ACACA and ACACB genes: 5'-TTTGGGGATCTCTAGCCTAC-3' and 5'-TAGGGAGTTTCTCCGCCGAC-3', respectively. The protocol used for generating ACC1 and ACC2 knockouts in BLaER1 cells is described in detail by Vierbuchen et al. (36). Clones with frameshift mutations on both alleles (identified indels for ACC1-KO and ACC2-KO cells were $-7 /+1$ and $-1 /+2$, respectively) were identified using the Tracking of Indels by Decomposition (TIDE) online software (75) and used for further experiments.

Transdifferentiation of wild-type, ACC1-KO, and ACC2-KO BLaER1 cells into macrophages was induced as previously described (36). BLaER1 macrophages were then seeded onto coated (natural mussel adhesive protein, Abcam) culture plates (Thermo Fisher Scientific), and cells were incubated in cell culture medium in the absence of IL-3 and $\beta$-estradiol overnight.

RT-qPCR. Total RNA of cells lysed in Trizol (peqGOLD TriFast, VWR International) was extracted by use of DirectZol RNA MiniPrep (Zymo Research) according to the manufacturer's instructions. For reverse transcription of RNA, the Maxima First Strand cDNA Synthesis Kit for RT-qPCR (Thermo Fisher Scientific) was used. RT-qPCR was performed using the LightCycler 480 Probe Master Kit and the LightCycler 480 II system (Roche) as described previously (76). Further details on primer pairs and probes used are provided in Supplemental Methods.

Microarray analyses. Integrity of extracted, total RNA was analyzed with the RNA Nano 6000 Kit on a Bioanalyzer (Agilent) according to the manufacturer's instructions. Total RNA was used for reverse amplification and Cy3 labeling of cRNA as well as hybridization on Agilent Mouse Whole Genome 4x44K V2 arrays, and scanning was conducted as described elsewhere (77). GeneSpring version 12.6 (Agilent) was used for analysis of data with removal of compromised probes prior to analysis. Differences in gene expression were computed using a moderated $t$ test with a Benjamini-Hochberg multiple-comparison correction cutoff of $P \leq 0.05$ between infected $W n t 6^{+/+}$and $W n t 6^{-/-}$macrophages. Gene symbols of significantly regulated genes were used to query the Molecular Signatures Database version 6.0 (http://software.broadinstitute. org/gsea/msigdb) for enrichment of Reactome gene sets with an FDR $q$ value cutoff of $P \leq 0.05$. The data set has been deposited in the NCBI's Gene Expression Omnibus database (accession number GSE160039).
Histology and immunohistochemistry. Lung tissue from patients with a multidrug-resistant TB was surgically removed (University Hospital Schleswig-Holstein [UKSH], Lübeck, Germany), dissected, and fixed with $10 \%$ formalin for $24-48$ hours. For immunohistochemical stainings, 1- to 2- $\mu \mathrm{m}$ sections were incubated in Antibody Diluent (Zytomed Systems) in the presence of a primary antibody specific for WNT6 (5 $\mu \mathrm{g} / \mathrm{mL}$, ab50030, Abcam; or $6.6 \mu \mathrm{g} / \mathrm{mL}, \mathrm{AF} 4109$, Bio-Techne), CD68 (1:100; clone PG-M1, Agilent), PLIN2 (1:100; ab78920, Abcam), ACC2 (LS-C11360, LSBio), and ACC1/2 (mAb, C83B10, Cell Signaling Technology). If necessary, tissue slides were incubated in Antibody Diluent (Zytomed Systems) containing a specific secondary antibody $\left[\mathrm{F}\left(\mathrm{ab}^{\prime}\right)_{2}\right.$ fragment rabbit anti-sheep, Jackson ImmunoResearch Laboratories; or rabbit anti-mouse IgG, Zytomed Systems; both 1:500 in Antibody Diluent) for 30-60 minutes. For detection and visualization, an HRP-conjugated polymer-based detection system (ZytoChem Plus Kit anti-rabbit) and chromogene 3-amino-9-ethylcarbazole (AEC) (both from Zytomed Systems) were used according to the manufacturer's instructions. To assess the histopathology in the lungs and livers of Mtb-infected mice, images of hematoxylin- or hematoxylin and eosin-stained tissue sections were acquired with a light microscope (BX41, Olympus) and assembled by real-time image stitching with CellSens standard software version 2.1 (Olympus). From each liver, a section of the median and tail lobe was analyzed, resulting in a total analyzed liver area of at least $4 \times 10^{7} \mu \mathrm{m}^{2}$ per mouse. Infiltrates were manually identified by their nuclear appearance, and the size of the infiltrates was determined by use of CellSens standard software (Olympus). To visualize lipid droplets by light microscopy, frozen lung tissue sections $(5 \mu \mathrm{m})$ were fixed (10\% [vol/vol] ice-cold formalin), washed with 60\% 2-propanol (MilliporeSigma), and incubated in Oil Red O solution (MilliporeSigma; 20 minutes). All slides were counterstained with Gill's hematoxylin (Vector Laboratories) and analyzed with a BX41 microscope (Olympus) and NIS-Elements software (NIS-Elements D3.10, SP3, Nikon).

Immunofluorescence and flow cytometry analyses. To monitor acidification of bacteria-containing compartments, macrophages infected with GFP-expressing Mtb were incubated with 400 nM LysoTracker dye (DND-99, Thermo Fisher Scientific) for 2 hours, thoroughly washed, fixed with $1 \%$ (wt/vol) paraformaldehyde, and incubated in PBS containing 10\% normal serum (PAN-Biotech) and 0.2\% Triton $\mathrm{X}$-100. For quantification of acidified, LysoTracker-positive compartments, samples were evaluated in a blinded fashion (counting of $>300$ phagosomes per condition). Lipid droplets and nuclei were stained with 4,4-difluoro-1,3,5,7,8-pentamethyl-4-bora-3a,4a-diaza-s-indacene (BODIPY 493/503; 5-10 $\mu \mathrm{g} / \mathrm{mL}$; Thermo Fisher Scientific) and DAPI $(1 \mu \mathrm{g} / \mathrm{mL}$; Roche). To compare the neutral lipid content of cells by fluorescence microscopy, images of BODIPY- and DAPI-stained peritoneal macrophages were acquired and analyzed with ImageJ software (version 1.51n, NIH) using a macro-script. Further details on this analysis are provided in Supplemental Methods. To visualize WNT6 and neutral lipids in tissue sections, frozen 5 - $\mu \mathrm{m}$-thick lung sections were fixed in $10 \%$ (vol/vol) ice-cold formalin and incubated sequentially with an antibody specific for WNT6 (6.6 $\mu \mathrm{g} / \mathrm{mL}$; AF4109, Bio-Techne) and a suitable Cy3-labeled secondary antibody (Minimal Cross Reactions, Jackson ImmunoResearch Laboratories) for 2 hours and 1 hour, respectively. Subsequently, neutral lipids and nuclei were visualized by use of BODIPY and DAPI, respectively. All slides were mounted with ProLong Antifade Reagents (Thermo Fisher Scientific) and analyzed by use of an Axio Observer microscope, equipped with an ApoTome, and Axio- 
Vision Software 4.8 or earlier (Carl Zeiss). To quantify neutral lipids by flow cytometry, detached NIH 3T3 cells were stained with BODIPY, washed, resuspended in PBS containing 0.2\% EDTA, and subjected to a MACS Quant Analyzer 10 (Miltenyi Biotec) using Miltenyi MACSQuantify software (version 2.6 or 2.8). To determine mitochondrial activity, BLaER1 cells or hMDMs were stained with the membrane potentialsensitive dye rhodamine 123 (25 minutes, $0.5 \mu \mathrm{g} / \mathrm{mL}$ ) and the membrane potential-independent dye MitoTracker Deep Red FM (300 nM) (both Thermo Fisher Scientific) to measure the mitochondrial activity and mitochondrial mass, respectively. Cells were washed once and immediately analyzed on the FACSCanto II (Becton Dickinson) using the BD FACSDiva software. In both cases, flow cytometry data were analyzed with FCS Express version 7 or earlier (De Novo Software).

Sample preparation for lipidomics. NIH 3T3 cells were detached, washed with PBS, and centrifuged $(10,000 \mathrm{~g})$. BMDMs were incubated in the presence of fatty acids or appropriate controls, washed, detached on ice, and centrifuged. In both cases, the dry pellets were immediately stored at $-80^{\circ} \mathrm{C}$ until lipids were extracted. Mouse lungs were homogenized in PBS containing Protease Inhibitor Cocktail (Protean, Roche), incubated in methanol ( $\geq 99 \%$ Chromasolv, Merck), and stored at $-80^{\circ} \mathrm{C}$ until lipid extraction.

Isotopic labeling experiments. Uniformly ${ }^{13} \mathrm{C}$-labeled oleic acid $\left(\mathrm{U}^{-13} \mathrm{C}_{18}, 98 \%\right.$, Cambridge Isotope Laboratories) was solubilized in ethanol (pure, AppliChem) and conjugated to BSA $\left({ }^{13} \mathrm{C}\right.$-oleate-BSA) as described above. Monocytes were incubated for 7 days with 200 $\mu \mathrm{M}{ }^{13} \mathrm{C}$-oleate-BSA during differentiation into macrophages. Subsequently, after culture media exchange, hMDMs were infected with Mtb (MOI 1:1) for 4 hours. After removal of extracellular bacteria, cells were incubated in the absence and presence of ACC 2 inhibitor 3 for 7 days. Finally, cells were detached on ice, washed, incubated in methanol, and stored at $-80^{\circ} \mathrm{C}$ until lipid extraction.

Lipid extraction and lipidomics. Total lipids were extracted according to a customized methyl-tert-butyl ether (MTBE) method (78). For further details on the customized protocols, see Supplemental Methods. Shotgun lipidomics measurements were performed using a $\mathrm{Q}$ Exactive (Thermo Fisher Scientific) or an Apex Qe Fourier Transform Ion Cyclotron Resonance mass spectrometer (Bruker Daltonik), both equipped with a TriVersa NanoMate (Advion BioSciences) as autosampler and ion source $(78,79)$. Lipid identification was performed using LipidXplorer (80), and quantitation was achieved in reference to a mix of internal standards, which were added before extraction. Abundance (Mol \%) of a given lipid is defined as the amount of a constituent (expressed in moles) divided by the total amount of all lipid constituents detected (also expressed in moles) multiplied by 100. Further information is provided in Supplemental Methods.

Cell viability assay. Real-time impedance measurements were conducted on an xCELLigence System (ACEA Biosciences) using plates with incorporated sensor array (E-Plate) and the Real-Time Cell Analyzer SP instrument. Data obtained were analyzed using Real-Time Cell Analyzer Software 1.2 (ACEA Biosciences).

Nitrite and cytokine quantification. To determine the production of reactive nitrogen intermediates, nitrite levels in supernatants of in vitro-cultivated cells were quantified using the Griess test as described in detail previously (81).

To determine cytokine levels in Mtb-infected mouse lungs, homogenates were analyzed with a bead-based assay panel (Mouse Proinflammatory Chemokine and Mouse Inflammation Panels, LEG-
ENDplex, BioLegend) according to the manufacturer's instructions. Measurements were performed on a FACSCanto II (Becton Dickinson) flow cytometer, and data were analyzed using FCAP Array Software version 3.0 (Becton Dickinson).

Extracellular flux analysis. BMDMs were incubated for 24 hours in the absence (control, BSA) or presence of oleic acid (oleate-BSA, 200 $\mu \mathrm{M})$, washed with XF DMEM (pH 7.4, 25 mM D-glucose [Carl Roth] and $1 \mathrm{mM}$ pyruvate [Merck]), incubated $\left(1 \mathrm{hour}, 37^{\circ} \mathrm{C}\right.$ ), and analyzed in an XF24 Extracellular Flux Analyzer (Agilent). During measurements, oligomycin $(1 \mu \mathrm{M})$, FCCP [carbonyl cyanide 4-(trifluoromethoxy) phenylhydrazone; $1.5 \mu \mathrm{M}$ ), and rotenone/antimycin $\mathrm{A}(1 \mu \mathrm{M})$ (all from Agilent) were injected. Data were analyzed by use of Seahorse XF24 software version 1.8.1.1.

Statistics. Statistical analyses were performed using GraphPad Prism 7 or earlier software versions (GraphPad Software). For statistical analyses of in vitro experiments, data were log-transformed in order to assume parametric distribution (82). For group comparison, a repeated-measures 1-way ANOVA followed by Holm-Šidák's multiple comparison as post hoc test was performed. For statistical analysis of in vivo experiments, data were tested for normality, log-transformed, and analyzed by an unpaired, 1-tailed (83) Student's $t$ test. A $P$ value below 0.05 was considered significant $\left({ }^{*} P \leq 0.05,{ }^{* *} P \leq 0.01,{ }^{* *} P \leq\right.$ 0.001). If not indicated otherwise, data are shown as mean \pm SEM.

Study approval. All experiments performed with primary human cells or human lung tissue were reviewed and approved by the Ethics Committee of the University of Lübeck (14-032, 12-220, 14-225, 18-194). All animal experiments were performed according to the German animal protection laws and were approved by the Animal Research Ethics Board of the Ministry of Environment (Kiel, Germany).

\section{Author contributions}

J Brandenburg, NR, JR, TG, UES, HH, CH, and DS planned and supervised experiments. J Brandenburg, SM, SCT, FW, TS, AG, SG, AH, LL, MH, and J Behrends conducted experiments. J Brandenburg, SM, SCT, FW, TV, TS, JR, AG, SG, AH, MH, MR, J Behrends, $\mathrm{CH}, \mathrm{SN}$, DS, and NR analyzed the data. All authors commented on the data. J Brandenburg and NR wrote the manuscript with input from all authors.

\section{Acknowledgments}

The authors are very grateful for funding by the German Research Foundation (DFG) within the Priority Program (DFG SPP1580) (Re1228 5-1 and Re1228 5-2 to NR) and the Cluster of Excellence 306 ("Inflammation at interfaces"), as well as for funding by the German Federal Ministry of Education (BMBF) via the German Center for Infection Research (DZIF) within the "Thematic translational unit tuberculosis" (TTU TB; TTU 02.705 to $\mathrm{CH}$; TTU 02.806 and 02.810 to NR; TTU 02.704-1 and 02.811 to DS). Moreover, we gratefully acknowledge Carolin Golin, Lisa Niwinski, and Johanna Volz for expert technical assistance. Finally, we thank Stefan Ehlers for critically reviewing the manuscript and making valuable suggestions for its improvement.

Address correspondence to: Norbert Reiling, Microbial Interface Biology, Research Center Borstel, Leibniz Lung Center, Parkallee 1-40, 23845 Borstel, Germany. Phone: 49.4537.188.4860; Email: nreiling@fz-borstel.de. 
1. World Health Organization. Global tuberculosis report 2020. https://apps.who.int/iris/bitstream/ handle/10665/336069/9789240013131-eng. pdf. Accessed July 7, 2021.

2. Dheda K, et al. The epidemiology, pathogenesis, transmission, diagnosis, and management of multidrug-resistant, extensively drug-resistant, and incurable tuberculosis. Lancet Respir Med. 2017;5(4):291-360.

3. Wallis RS, Hafner R. Advancing host-directed therapy for tuberculosis. Nat Rev Immunol. 2015;15(4):255-263.

4. Reiling N, et al. Shaping the niche in macrophages: genetic diversity of the M. tuberculosis complex and its consequences for the infected host. Int J Med Microbiol. 2018;308(1):118-128.

5. Prosser G, et al. The bacillary and macrophage response to hypoxia in tuberculosis and the consequences for $\mathrm{T}$ cell antigen recognition. Microbes Infect. 2017;19(3):177-192.

6. Rodríguez-Prados J-C, et al. Substrate fate in activated macrophages: a comparison between innate, classic, and alternative activation. J Immunol. 2010;185(1):605-614.

7. Shi L, et al. Infection with Mycobacterium tuberculosis induces the Warburg effect in mouse lungs. Sci Rep. 2015;5:18176.

8. Tannahill GM, et al. Succinate is an inflammatory signal that induces IL-1 $\beta$ through HIF-1 $\alpha$. Nature. 2013;496(7444):238-242.

9. Jha AK, et al. Network integration of parallel metabolic and transcriptional data reveals metabolic modules that regulate macrophage polarization. Immunity. 2015;42(3):419-430.

10. Gleeson LE, et al. Cutting edge: Mycobacterium tuberculosis induces aerobic glycolysis in human alveolar macrophages that is required for control of intracellular bacillary replication. J Immunol. 2016;196(6):2444-2449.

11. Huang L, et al. Growth of Mycobacterium tuberculosis in vivo segregates with host macrophage metabolism and ontogeny. J Exp Med. 2018;215(4):1135-1152.

12. Huang Y, et al. Toll-like receptor agonists promote prolonged triglyceride storage in macrophages. J Biol Chem. 2014;289(5):3001-3012.

13. Boström P, et al. Hypoxia converts human macrophages into triglyceride-loaded foam cells. Arterioscler Thromb Vasc Biol. 2006;26(8):1871-1876.

14. Russell DG, et al. Foamy macrophages and the progression of the human tuberculosis granuloma. Nat Immunol. 2009;10(9):943-948.

15. Peyron P, et al. Foamy macrophages from tuberculous patients' granulomas constitute a nutrient-rich reservoir for M. tuberculosis persistence. PLoS Pathog. 2008;4(11):e1000204.

16. Muñoz-Elías EJ, McKinney JD. Mycobacterium tuberculosis isocitrate lyases 1 and 2 are jointly required for in vivo growth and virulence. $\mathrm{Nat}$ Med. 2005;11(6):638-644.

17. Pandey AK, Sassetti CM. Mycobacterial persistence requires the utilization of host cholesterol. Proc Natl Acad Sci U S A. 2008;105(11):4376-4380.

18. Willert K, Nusse R. Wnt proteins. Cold Spring Harb Perspect Biol. 2012;4(9):a007864.

19. Brandenburg J, Reiling N. The Wnt blows: on the functional role of Wnt signaling in Mycobacterium tuberculosis infection and beyond. Front
Immunol. 2016;7:635.

20. Schaale $\mathrm{K}$, et al. Wnt6 is expressed in granulomatous lesions of Mycobacterium tuberculosis-infected mice and is involved in macrophage differentiation and proliferation. J Immunol. 2013;191(10):5182-5195.

21. Heitmann $\mathrm{L}$, et al. The IL-13/IL- $4 \mathrm{R} \alpha$ axis is involved in tuberculosis-associated pathology. J Pathol. 2014;234(3):338-350.

22. Listenberger LL, Brown DA. Fluorescent detection of lipid droplets and associated proteins. Curr Protoc Cell Biol. 2007; Chapter 24:Unit 24.2.

23. Holla S, et al. MUSASHI-mediated expression of JMJD3, a H3K27me3 demethylase, is involved in foamy macrophage generation during Mycobacterial infection. PLoS Pathog. 2016;12(8):e1005814.

24. Singh V, et al. Mycobacterium tuberculosisdriven targeted recalibration of macrophage lipid homeostasis promotes the foamy phenotype. Cell Host Microbe. 2012;12(5):669-681.

25. Dkhar HK, et al. Mycobacterium tuberculosis keto-mycolic acid and macrophage nuclear receptor TR4 modulate foamy biogenesis in granulomas: a case of a heterologous and noncanonical ligand-receptor pair. J Immunol. 2014;193(1):295-305.

26. Subramanian A, et al. Gene set enrichment analysis: a knowledge-based approach for interpreting genome-wide expression profiles. Proc Natl Acad Sci U S A. 2005;102(43):15545-15550.

27. Pepino MY, et al. Structure-function of CD36 and importance of fatty acid signal transduction in fat metabolism. Annu Rev Nutr. 2014;34:281-303.

28. Bu SY, Mashek DG. Hepatic long-chain acyl-CoA synthetase 5 mediates fatty acid channeling between anabolic and catabolic pathways. J Lipid Res. 2010;51(11):3270-3280.

29. Wanders RJA, et al. The enzymology of mitochondrial fatty acid beta-oxidation and its application to follow-up analysis of positive neonatal screening results. J Inherit Metab Dis. 2010;33(5):479-494.

30. Hunt MC, et al. The emerging role of acyl-CoA thioesterases and acyltransferases in regulating peroxisomal lipid metabolism. Biochim Biophys Acta. 2012;1822(9):1397-1410.

31. Harris CA, et al. DGAT enzymes are required for triacylglycerol synthesis and lipid droplets in adipocytes. JLipid Res. 2011;52(4):657-667.

32. Bulankina AV, et al. TIP47 functions in the biogenesis of lipid droplets. J Cell Biol. 2009;185(4):641-655.

33. Kim C-W, et al. Acetyl CoA carboxylase inhibition reduces hepatic steatosis but elevates plasma triglycerides in mice and humans: a bedside to bench investigation. Cell Metab. 2017;26(2):394-406.

34. Abu-Elheiga L, et al. Continuous fatty acid oxidation and reduced fat storage in mice lacking acetyl-CoA carboxylase 2 . Science. 2001;291(5513):2613-2616.

35. Rapino F, et al. C/EBP $\alpha$ induces highly efficient macrophage transdifferentiation of $\mathrm{B}$ lymphoma and leukemia cell lines and impairs their tumorigenicity. Cell Rep. 2013;3(4):1153-1163.

36. Vierbuchen T, et al. The human-associated archaeon Methanosphaera stadtmanae is recognized through its RNA and induces TLR8- dependent NLRP3 inflammasome activation. Front Immunol. 2017;8:1535.

37. Daniel J, et al. Mycobacterium tuberculosis uses host triacylglycerol to accumulate lipid droplets and acquires a dormancy-like phenotype in lipid-loaded macrophages. PLoS Pathog. 2011;7(6):e1002093.

38. Lee W, et al. Intracellular Mycobacterium tuberculosis exploits host-derived fatty acids to limit metabolic stress. JBiol Chem. 2013;288(10):6788-6800.

39. Meena LS, Kolattukudy PE. Expression and characterization of Rv0447c product, potentially the methyltransferase involved in tuberculostearic acid biosynthesis in Mycobacterium tuberculosis. Biotechnol Appl Biochem. 2013;60 (4):412-416.

40. Brandenburg J, et al. Tuberculostearic acid (TSA)-containing phosphatidylinositols as reliable marker to determine Mycobacterium tuberculosis bacterial burden [preprint]. https:// doi.org/10.1101/2021.02.04.429149. Posted on bioRxiv April 26, 2021.

41. Choi CS, et al. Continuous fat oxidation in acetylCoA carboxylase 2 knockout mice increases total energy expenditure, reduces fat mass, and improves insulin sensitivity. Proc Natl Acad Sci US A. 2007;104(42):16480-16485.

42. Follstad BD, et al. Mitochondrial membrane potential differentiates cells resistant to apoptosis in hybridoma cultures. Eur J Biochem. 2000;267(22):6534-6540.

43. Kampe K, et al. Susceptibility of podocytes to palmitic acid is regulated by fatty acid oxidation and inversely depends on acetyl-CoA carboxylases 1 and 2. Am J Physiol Renal Physiol. 2014;306(4):F401-F409.

44. Chan FK-M, et al. Detection of necrosis by release of lactate dehydrogenase activity. Methods Mol Biol. 2013;979:65-70.

45. Dorhoi A, et al. Type I IFN signaling triggers immunopathology in tuberculosis-susceptible mice by modulating lung phagocyte dynamics. Eur J Immunol. 2014;44(8):2380-2393.

46. Svensson RU, et al. Inhibition of acetyl-CoA carboxylase suppresses fatty acid synthesis and tumor growth of non-small-cell lung cancer in preclinical models. Nat Med. 2016;22(10):1108-1119.

47. Loomba R, et al. GS-0976 reduces hepatic steatosis and fibrosis markers in patients with nonalcoholic fatty liver disease. Gastroenterology. 2018;155(5):1463-1473.

48. Lawitz EJ, et al. Acetyl-CoA carboxylase inhibitor GS-0976 for 12 weeks reduces hepatic de novo lipogenesis and steatosis in patients with nonalcoholic steatohepatitis. Clin Gastroenterol Hepatol. 2018;16(12):1983-1991.

49. Scott CC, et al. TFAP2 transcription factors are regulators of lipid droplet biogenesis. Elife. 2018;7:e36330.

50. Go G-W, et al. The combined hyperlipidemia caused by impaired Wnt-LRP6 signaling is reversed by Wnt3a rescue. Cell Metab. 2014;19(2):209-220.

51. Zhao F, et al. Paracrine Wnt5a- $\beta$-catenin signaling triggers a metabolic program that drives dendritic cell tolerization. Immunity. 2018;48(1):147-160.

52. Fuster JJ, et al. Noncanonical Wnt signaling promotes obesity-induced adipose tissue 
inflammation and metabolic dysfunction independent of adipose tissue expansion. Diabetes. 2015;64(4):1235-1248.

53. You J, et al. Wnt pathway-related gene expression in inflammatory bowel disease. Dig Dis Sci. 2008;53(4):1013-1019.

54. Choy DF, et al. Gene expression patterns of Th2 inflammation and intercellular communication in asthmatic airways. JImmunol. 2011;186(3):1861-1869.

55. Melo RCN, Dvorak AM. Lipid body-phagosome interaction in macrophages during infectious diseases: host defense or pathogen survival strategy? PLoS Pathog. 2012;8(7):e1002729.

56. Feingold KR, et al. ADRP/ADFP and Mal1 expression are increased in macrophages treated with TLR agonists. Atherosclerosis. 2010;209(1):81-88.

57. Feingold KR, et al. Mechanisms of triglyceride accumulation in activated macrophages. J Leukoc Biol. 2012;92(4):829-839.

58. Ouimet M, et al. Mycobacterium tuberculosis induces the miR-33 locus to reprogram autophagy and host lipid metabolism. Nat Immunol. 2016;17(6):677-686.

59. Nazarova EV, et al. Rv3723/LucA coordinates fatty acid and cholesterol uptake in Mycobacterium tuberculosis. Elife. 2017;6:e26969.

60. Nazarova EV, et al. The genetic requirements of fatty acid import by Mycobacterium tuberculosis within macrophages. Elife. 2019;8:e43621.

61. den Brok MH, et al. Lipid droplets as immune modulators in myeloid cells. Trends Immunol. 2018;39(5):380-392.

62. Knight M, et al. Lipid droplet formation in Mycobacterium tuberculosis infected macrophages requires IFN- $\gamma /$ HIF- $1 \alpha$ signaling and supports host defense. PLoS Pathog. 2018;14(1):e1006874. 63. de Mello ES, Ferreira Alves VA. Hepatic gran- ulomas: differential diagnosis. In: Saxena R, ed. Practical Hepatic Pathology: A Diagnostic Approach. 2nd ed. Elsevier; 2018:289-300.

64. Hickey AJ, et al. A systematic review of hepatic tuberculosis with considerations in human immunodeficiency virus co-infection. BMC Infect Dis. 2015;15:209.

65. Pond SM, Tozer TN. First-pass elimination. Basic concepts and clinical consequences. Clin Pharmacokinet. 1984;9(1):1-25.

66. Fallon PG, et al. IL-13 overexpression predisposes to anaphylaxis following antigen sensitization. JImmunol. 2001;166(4):2712-2716.

67. Wang $\mathrm{Q}$, et al. Wnt6 is essential for stromal cell proliferation during decidualization in mice. Biol Reprod. 2013;88(1):5.

68. Steinhäuser C, et al. Immunomagnetic isolation of pathogen-containing phagosomes and apoptotic blebs from primary phagocytes. Curr Protoc Immunol. 2014;105:14.36.1-14.36.26.

69. Zhang $X$, et al. The isolation and characterization of murine macrophages. Curr Protoc Immunol. 2008; Chapter 14:Unit 14.1.

70. Reiling N, et al. Clade-specific virulence patterns of Mycobacterium tuberculosis complex strains in human primary macrophages and aerogenically infected mice. mBio. 2013;4(4):e00250-13.

71. Song $\mathrm{H}$, et al. Identification of outer membrane proteins of Mycobacterium tuberculosis. Tuberculosis (Edinb). 2008;88(6):526-544.

72. Kolbe K, et al. Azido pentoses: a new tool to efficiently label Mycobacterium tuberculosis clinical isolates. Chembiochem. 2017;18(13):1172-1176.

73. Zelmer A, et al. A new in vivo model to test anti-tuberculosis drugs using fluorescence imaging. J Antimicrob Chemother. 2012;67(8):1948-1960.

74. Reiling N, et al. Cutting edge: Toll-like recep- tor (TLR)2- and TLR4-mediated pathogen recognition in resistance to airborne infection with Mycobacterium tuberculosis. JImmunol. 2002;169(7):3480-3484.

75. Brinkman EK, et al. Easy quantitative assessment of genome editing by sequence trace decomposition. Nucleic Acids Res. 2014;42(22):e168.

76. Neumann J, et al. Frizzled 1 is a marker of inflammatory macrophages, and its ligand Wnt3a is involved in reprogramming Mycobacterium tuberculosis-infected macrophages. FASEB J. 2010;24(11):4599-4612.

77. Marwitz S, et al. Downregulation of the TGF $\beta$ pseudoreceptor BAMBI in non-small cell lung cancer enhances TGF $\beta$ signaling and invasion. Cancer Res. 2016;76(13):3785-3801.

78. Eggers LF, Schwudke D. Shotgun lipidomics approach for clinical samples. Methods Mol Biol. 2018;1730:163-174.

79. Graessler J, et al. Top-down lipidomics reveals ether lipid deficiency in blood plasma of hypertensive patients. PLoS One. 2009;4(7):e6261.

80. Herzog R, et al. A novel informatics concept for high-throughput shotgun lipidomics based on the molecular fragmentation query language. Genome Biol. 2011;12(1):R8.

81. Hölscher C, et al. Defective nitric oxide effector functions lead to extreme susceptibility of trypanosoma cruzi-infected mice deficient in gamma interferon receptor or inducible nitric oxide synthase. Infect Immun. 1998;66(3):1208-1215.

82. Willems E, et al. Standardization of real-time PCR gene expression data from independent biological replicates. Anal Biochem. 2008;379(1):127-129.

83. Jones LV, Tukey JW. A sensible formulation of the significance test. Psychol Methods. 2000;5(4):411-414. 